\title{
Privacy-Aware Laser Wireless Power Transfer for Aerial Multi-Access Edge Computing: A Colonel Blotto Game Approach
}

\author{
Long Zhang, Member, IEEE, Yao Wang, Minghui Min, Member, IEEE, Chao Guo, \\ Vishal Sharma, Member, IEEE, and Zhu Han, Fellow, IEEE
}

\begin{abstract}
Multi-access edge computing (MEC) has been recently considered in challenging environments lacking available terrestrial infrastructures by extending the computing resources to the air for further enhancing the computation capability of the new aerial user equipment (AUE). Additionally, wireless power transfer (WPT) is a promising solution to prolong the battery lifetime of energy-constrained wireless devices like AUEs. In this paper, we investigate the integration of laser-beamed WPT in the high-altitude platform (HAP) aided MEC systems for the HAP-connected AUEs. By discretizing the three-dimensional coverage space of the HAP, we present a multi-tier tile grid-based spatial structure to provide aerial locations for laser charging. With this setup, we identify a new privacy vulnerability caused by the openness during the air-to-air transmission of WPT signaling messages in the presence of a terrestrial adversary. A privacy-aware laser-powered aerial MEC framework is developed that addresses this vulnerability and enhances the location privacy of AUEs for laser WPT. Specifically, the interaction between the HAP as a defender and the adversary in their tile grid allocation as charging locations to AUEs is formulated as a Colonel Blotto game, which models the competition of the players for limited resources over multiple battlefields for a finite time horizon. Moreover, we derive the mixed-strategy Nash equilibria of the tile grid allocation game for both symmetric and asymmetric tile grids between the defender and the adversary. Simulations results show that the proposed framework significantly outperforms the design baselines with a given privacy protection level in terms of system-wide expected total utilities.
\end{abstract}

Index Terms-Multi-access edge computing, wireless power transfer, laser power transfer, privacy, high-altitude platform, aerial user equipment, tile grid allocation, Colonel Blotto game.

\section{INTRODUCTION}

W ITH the recent advancements in the fifth generation (5G) enabling technologies and the Internet of Things (IoT), the phenomenal growth of smart user equipment (UE) has been driving the rapid development of numerous attractive mobile applications and multimedia services, such as extended reality, holographic telepresence, face recognition, autonomous driving, etc. In addition to the classical ground UEs, unmanned aerial vehicles (UAVs) can act as the new aerial UEs (AUEs), which are also referred to as the cellularconnected drone users [2]. The difference of the AUEs with respect to the terrestrial UEs is their ability to intelligently

- L. Zhang and Y. Wang are with the School of Information and Electrical Engineering, Hebei University of Engineering, Handan 056038, China. E-mail: zhanglong@hebeu.edu.cn, yaow0518@gmail.com

- M. Min is with the School of Information and Control Engineering, China University of Mining and Technology, Xuzhou 221116, China. E-mail: minminghuismile@gmail.com

- C. Guo is with the Department of Communication Engineering, Beijing Electronics Science and Technology Institute, Beijing 100070, China. E-mail:guochao@besti.edu.cn

- V. Sharma is with the School of Electronics, Electrical Engineering and Computer Science, Queens University Belfast, Northern Ireland, UK. E-mail:V.Sharma@qub.ac.uk

- Z. Han is with the Department of Electrical and Computer Engineering, University of Houston, Houston, TX 77004, USA, and also with the Department of Computer Science and Engineering, Kyung Hee University, Seoul 446-701, South Korea.

E-mail:zhan2@uh.edu

The material in this paper was presented in part at the IEEE GLOBECOM, Taipei, Taiwan, Dec. 2020 [1]. fly in the three-dimensional (3D) space and flexibly optimize their trajectory to complete their missions.

Thanks to their in-built properties, the use of AUEs has raised a great upsurge of interest recently for both civilian and commercial applications, e.g., aerial surveillance, flying cars, disaster response, etc $[3]-[5]$. Among them, most of the applications are not only computation-intensive but also latency-sensitive. For instance, a huge amount of data collected by the AUEs in the missions of disaster sensing, e.g., situational awareness, mapping, and reconnaissance, generally consume extensive computational resources [6]. However, the constrained computing capability of the AUEs is usually difficult to fulfill the computation requirements of these applications. To tackle this issue, multi-access edge computing (MEC) is envisioned as a promising approach to provide the cloud-computing capability in close proximity to the users [7|. By enabling the users to offload their computation tasks to the network edges for execution, MEC has the potential to bring many advantages including reduced latency, enhanced security, improved energy saving, enhanced user experience, etc.

To provide the edge computing resources for the AUEs, one general solution is to integrate the MEC servers at the edge of terrestrial networks, e.g., gNBs in 5G wireless systems. However, it is impractical and uneconomical to deploy such a kind of terrestrial infrastructure integrated with MEC servers for the AUEs when they are distributed above the challenging hard-to-reach environments, e.g., dis- 
aster scenarios and ocean areas. An alternative is to extend the computation resources to the air for providing efficient and flexible computing services for the AUEs. Compared to the UAVs in lower height above the earth, high-altitude platforms (HAPs) in the stratosphere are more suitable to offer elastic computing services because of larger area coverage, bigger payload capacity, and longer endurance [8]. To this end, the integration of the HAPs with MEC servers motivates the prospect of implementing the aerial computation offloading for the HAP-connected AUEs.

Although HAP-aided MEC can reduce energy consumption effectively, computation offloading may be still interrupted due to the limited energy storage (e.g., on-board lithium-ion polymer battery capacity) of the AUEs [9]. A promising solution is to apply the wireless power transfer (WPT) to provide a convenient, cost-effective, and environmentally friendly energy supply to charge the AUEs for energy replenishment. The existing WPT technologies commonly consist of near-field WPT and far-field WPT. The near-field WPT utilizes inductive coupling or magnetic resonance to transfer energy over very short distances from a few millimeters to several meters [10]. In comparison with the near-field methods, the far-field WPT has emerged as a prospective solution to the sustainability of future generation networks, due to the attractive advantages such as long charging ranges, highly mobility support, small form factors of the receiver, integration with wireless communications, etc [11]. Recently, laser-beamed far-field WPT is becoming a viable solution to provide a controllable, sustainable, and longer-range energy supply for extending the lifetime of the UAVs [12], [13], which can be also applicable for the energyconstrained AUEs.

Unlike the far-field WPT enabled by radio frequency (RF), the laser WPT is able to deliver much more energy to the users or the receivers with high energy concentration over long distances [12]. It has been demonstrated by the JAXA that an orbiting satellite with a solar condenser can quickly distribute tens of kilowatt (kW)-class laser-beamed energy on demand to the target areas over the Earth's surface [14]. With this in mind, we propose to employ the laser WPT for HAP-aided MEC to develop a completely novel paradigm of the laser-powered aerial MEC system, wherein a laser transmitter and MEC server are mounted at the HAP. With this setup, the HAP transmits laser-beamed energy to charge the AUEs from the sky, and the AUEs utilize the harvested energy to support the flights and complete the computation offloading.

In this context, to obtain an effective aerial location for laser charging, each AUE is required to transmit a WPT signaling message to the HAP on a control channel through the air-to-air (A2A) uplink transmission. However, the WPT signaling delivery on the control channel in an open radio environment is unattended and lacks protected physical boundaries, which makes the wireless communications vulnerable to many security threats from adversaries [15]. By actively sending the false charging location data to AUEs, an external adversary can launch the attacks of charging location data falsification (CLDF), resulting in the failure of charging operations. Essentially, the privacy of the AUEs' charging locations is also compromised by observing the WPT signaling dissemination at the adversary.
Consequently, enhanced security and privacy mechanisms are of paramount importance to the WPT since a minor compromise may result in a major security problem, e.g., the leakage of aerial charging location information associated with the AUEs. This, however, may allow the adversary to stealthily infer and track the aerial charging locations of the AUEs to launch the CLDF attacks for laser WPT. Such kinds of attacks may further lead to the unsuccessful laser WPT and the failure of aerial computation offloading. It is for this reason that enhanced privacy mechanism is a key issue in the field of laser WPT for HAP-aided MEC. Aiming to address the above privacy-associated problems, there are $\mathrm{t}$ wo key network bottlenecks that must be overcome, namely privacy-aware laser WPT to secure the aerial MEC systems as well as the impact of system performance tradeoff on the total utilities for aerial MEC. Admittedly, these bottlenecks and challenges motivate the need for a better understanding of the interplay between privacy preservation and system performance, which typically require a balance between them.

Motivated by the above observations, we can find that the exploration of privacy-aware laser WPT for the HAPaided aerial MEC systems has become highly valuable. Our objective in this paper is to achieve enhanced location privacy for AUEs through identifying such an interplay under the scenario of laser-powered aerial MEC. To the best of our knowledge, this is the first work that considers the aerial location privacy for laser WPT in the literature. For bridging the research gap, we investigate a privacy-aware laser WPT problem in this paper via the Colonel Blotto game framework for the laser-powered aerial MEC systems, aiming to reveal a tradeoff between privacy preservation and system performance. The main contributions of this paper can be summarized as follows.

- We propose a multi-tier grid-based spatial structure for charging the AUEs to discretize the 3D coverage space of the HAP in the laser-powered aerial MEC systems. This is a new approach towards the 3D spatial view of charging location design for AUEs in the sky by exploiting the equally sized square tile grids within a finite number of tiers.

- With the tile grid-dependent spatial structure, we develop a privacy-aware laser-powered aerial MEC framework, which not only achieves the enhanced location privacy for AUEs in the presence of a terrestrial external adversary but also accomplishes the aerial computation offloading from AUEs to the HAP. Our framework is the first time in the literature to identify a close coupling of laser WPT and privacy protection to secure the aerial MEC systems under the CLDF attacks mounted by the adversary.

- We formulate the competitive interaction between the HAP as a defender and the adversary in their tile grid allocations for the aerial charging locations to AUEs as a Colonel Blotto game, referred to as the tile grid allocation game. Particularly, the defender seeks to optimally allocate its defense resources regarding the legitimate tile grids over AUEs, while the adversary tries to assign its attack resources in terms of the false tile grids. Such kind of interaction 
over an attack-defense resource allocations models the competition of two players, i.e., the defender and the adversary, in this game framework for limited resources over a finite set of battlefields.

- We design the utility function that each player receives on an individual battlefield during a given time horizon by capturing the balance among the local computation capacity, the energy consumption, and the execution delay of each AUE. We accordingly formulate the total utilities obtained by the defender over the battlefield set. Then the mixed-strategy Nash equilibria of the tile grid allocation game are derived for both symmetric and asymmetric tile grids between the defender and the adversary. The mixed-strategy Nash equilibrium points are shown to evaluate how the system performance tradeoff for the laser-powered aerial MEC systems impacts the expected total utilities of the HAP.

The rest of this paper is organized as follows. In Section 2. we provide a review of related works. Section 3 describes the system model. In Section 4, we formulate the Colonel Blotto game between the defender and the adversary and discuss the solution of the game. Simulation results are presented in Section 5 . Finally, Section 6 concludes the paper.

\section{Related Works}

\subsection{Far-Field WPT in MEC Systems}

Recently, the integration of far-field WPT into MEC systems has received considerable attention, aiming to enhance the system performance by optimizing computation offloading and resource allocation jointly. In [16], the authors investigated the reward maximization problem to simultaneously maximize the data utility and minimize the energy consumption from the operators perspective, by jointly considering the wireless-power allocation at the base station (BS) as well as the offloaded data size and power allocation at the UEs. In [17], the authors explored the stochastic optimization problem to identify the tradeoff between timeaverage energy efficiency (EE) and delay for the multiple access-based wireless powered MEC systems, by optimizing the network EE under the energy causality and network stability constraints. The authors in [18] developed the optimization problem to minimize the total required energy of the UAV integrated with the MEC server, via jointly optimizing the computation bits, the CPU frequencies, the UE's transmit power, and the UAVs trajectory. However, these works mainly focused on the use of RF-based WPT at the terrestrial BS [16], |17| and the aerial infrastructure |18| to prolong the lifetime of energy-limited UEs on the ground.

\subsection{Laser WPT in Network Scenarios}

Several recent works have been devoted to the application of laser WPT as the energy supply in network scenarios for the energy-demanding devices, e.g., the UAVs [9], |12], |13], [19], [20] and the IoT devices [21]. Based on the downlink communication scenario for the laser-charged quadrotor $\mathrm{UAV}$, the authors in [9] established the relationship between the motion regimes, energy consumption/harvesting, and battery dynamics of the UAV. The cumulative downlink throughput maximization problem was proposed in [12] for the laser-powered UAV wireless systems by jointly optimizing the trajectory, velocity, acceleration, and transmit power of the UAV. In $|19|$, the authors analyzed the performance of simultaneous information and power transfer from the laser transmitter to the laser-powered UAV through power splitting and also derived the joint energy and the signal-tonoise ratio coverage probability via stochastic geometry. The authors in [13] devised the general optimization framework for joint power allocation and trajectory design in the UAVenabled mobile relaying system with laser charging. In [20], the authors employed the laser-driven adaptive WPT for the UAV-mounted relay-assisted IoT networks and formulated the optimization problem to maximize the number of the connected IoT devices for uplink data transmission. Compared with the studies applying laser WPT for the UAVs, the authors in [21] proposed to employ the distributed laser charging to provide the IoT devices with safe WPT capability with the aid of the photovoltaic cells instead of the collecting lens at the receiver. However, the above studies exploited the laser WPT only to the sscenarios of UAV communications and IoT systems and did not capture the effect of laser WPT on our considered HAP-aided MEC systems.

\subsection{Security and Privacy in WPT}

Despite the fact that the WPT-enabled techniques in energyconstrained wireless networks have been intensively studied in the recent literature, only a very few works consider the security and privacy issues related to the WPT itself. The authors in |15| summarized the primary security attacks arising from the lack of inherent security mechanisms in the WPT-enabled networks, and described the potential countermeasures to mitigate the attack effects. In [22], the authors designed the two-plane-based secure WPT architecture via the blockchain, and investigated the efficient WPT mechanism by combining the contract theory and the delegated proof-of-stake-based lightweight consensus scheme, respectively. Similar to [22], by utilizing the directed acyclic graph and consortium blockchain, the authors in [23 developed the distributed and secure UAV-aided WPT framework referred to as the aerial-ground chain, for protecting the UAVs as aerial energy transmitters against the energy attacks. In |24|, the authors exploited the chaotic energy encryption method to propose a secure inductive WPT system. Particularly, the energy of the power source was encrypted based on the specific chaotic map, and the decryption was conducted through the synchronized chaotic map. The above studies are heuristic, although they only investigated the impact of security attacks on the RF-based WPT process for the ground devices via enhancing the security mechanisms. By contrast, we extensively consider the privacy issues related to the laser WPT for the AUEs from the sky in the laser-powered aerial MEC systems.

\section{System MOdel}

\subsection{System Overview}

As shown in Fig. 1, we consider a laser-powered aerial MEC system, which consists of a multi-antenna HAP in- 


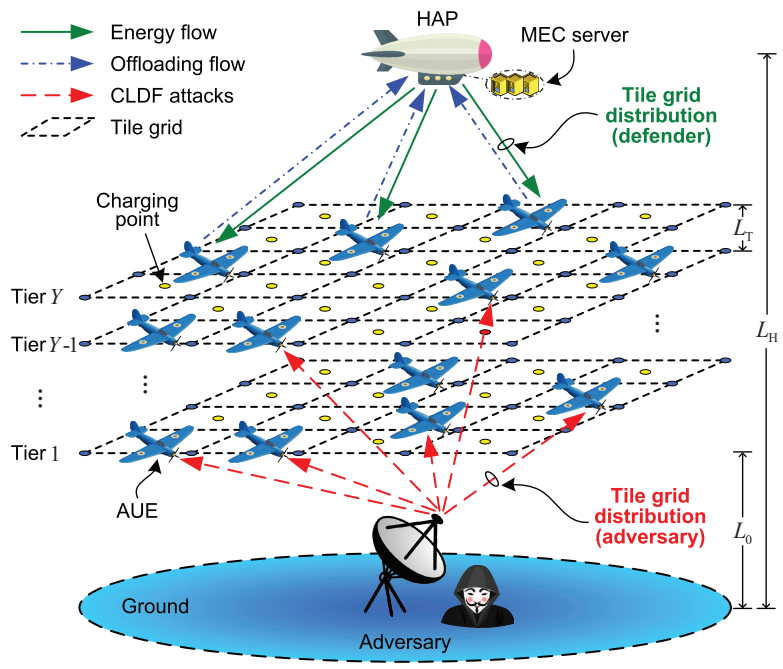

Fig. 1. Illustration of a laser-powered aerial MEC system consisting of an HAP and $N$ AUEs in the presence of an adversary on the ground.

tegrated with a laser transmitter and MEC server, and a set $\mathcal{N}=\{1,2, \cdots, N\}$ of single-antenna AUEs located inside the $3 \mathrm{D}$ coverage space $\Omega$ of the HAP, each having a computation-intensive latency-critical task to be completed. In this system, the HAP delivers laser-beamed energy to charge the AUEs in the sky over a long distance, and each AUE then uses the harvested energy to maintain the flight operation and accomplish the computation task via executing locally or offloading to the HAP. We, therefore, define the charging-then-MEC phase, which is undertaken over a finite time horizon with duration $T$. More particularly, the HAP is operating in a quasi-stationary position at a stratospheric altitude of $L_{\mathrm{H}}$ above the Earth's surface, which we assume to be constant and known at the HAP. Each AUE in a fixed-wing configuration is capable of intelligently flying and optimizing its trajectory in the HAP coverage of a 3D space $\Omega$. We further suppose that the cleared LoS with each AUE can be effectively established for the HAP due to its higher altitude operation and high-elevation angle.

Without loss of generality, the $3 \mathrm{D}$ coverage space $\Omega$ of interest is discretized into a multi-tier spatial structure consisting of equally sized square tile grids, as illustrated in Fig. 1. For the discretized spatial structure, we consider that there are $Y$ tiers and the lowest tier relative to the ground is referred to as tier 1 . We let $\mathcal{Y}=\{1,2, \cdots, Y\}$ denote the set of the tiers for such a structure, and define $L_{0}$ as the vertical distance between tier 1 and the Earth's surface. To simplify the analysis, we assume that every tier is composed of $Q \times Q$ square tile grids with equally sized areas, for $Q \times Q \times Y \gg N$, and all the tiers are evenly spaced by an equal vertical distance, denoted by $L_{\mathrm{T}}$, for $(Y-1) L_{\mathrm{T}} \ll L_{\mathrm{H}}$. Note that the tile grid in each tier is adopted as a spatial location to charge the AUE via the laser WPT from the HAP. Here, we focus on the center of the tile grid as the 3D location of the charging point for the AUE.

For ease of exposition, a 3D Cartesian coordinate system is adopted. The horizontal coordinate of the HAP can be determined by $\mathbf{q}^{\mathrm{H}}=\left[x^{\mathrm{H}}, y^{\mathrm{H}}\right]^{T} \in \mathbb{R}^{2 \times 1}$. During the charging-then-MEC phase within time horizon $T$, every
AUE is assumed to be flying at the fixed 3D location of charging point. Therefore, the horizontal coordinate of AUE $n$ in tier $\gamma$ during time horizon $T$ is formulated at $\mathbf{q}_{n}^{(\gamma)}=\left[x_{n}^{(\gamma)}, y_{n}^{(\gamma)}\right]^{T} \in \mathbb{R}^{2 \times 1}$, for $n \in \mathcal{N}$ and $\gamma \in \mathcal{Y}$. Accordingly, the vertical distance between AUE $n$ in tier $\gamma$ and the Earth's surface can be expressed as $d_{n}^{(\gamma)}=L_{0}+L_{\mathrm{T}}(\gamma-1)$. In what follows, unless otherwise stated, we use the terms "AUE $n$ in tier $\gamma$ " and "AUE $n$ " interchangeably. During time horizon $T$, the distance between the HAP and AUE $n$ in tier $\gamma$ is given by

$$
d_{n}^{(\gamma)}=\sqrt{\left[L_{\mathrm{H}}-\left(L_{0}+L_{\mathrm{T}}(\gamma-1)\right)\right]^{2}+\left\|\mathbf{q}^{\mathrm{H}}-\mathbf{q}_{n}^{(\gamma)}\right\|^{2}} .
$$

To obtain the laser-beamed power supply via the laser WPT in the air, each AUE needs to send a WPT signaling message to the HAP on a control channel via the A2A uplink transmission. When receiving the signaling message from the AUE, the HAP assigns a 3D location of charging point in terms of tile grid based on the discretized spatial structure, and then sends the charging location data to that AUE through the A2A downlink transmission. Thereby, we define the signaling transfer phase, the tile grid allocation phase, and the charging location determination phase, respectively. It is noted that the AUEs can obtain the results of tile grid allocation conducted by the HAP, i.e., the charging location data, during the charging location determination phase.

To facilitate the following analysis, we adopt a timeslotted frame structure, which is composed of four successive phases, namely, the signaling transfer phase, the tile grid allocation phase, the charging location determination phase, and the charging-then-MEC phase, as shown in Fig. 2. Particularly, we ignore the time of the WPT signaling transfer as the signaling overhead is usually of small data size [25]. Meanwhile, the time for the tile grid allocation at the HAP can be also neglected due to its high capabilities for decisionmaking, control, and processing. Moreover, we ignore the time for transmitting the charging location data to the AUEs during the charging location determination phase since the HAP has higher data throughput and small propagation delay [26], |27].

We adopt a time-switched "charging-then-MEC" strategy, and employ the different fractions of time for laser charging, task offloading, and result downloading. As such, the time horizon $T$ is equally divided into $K$ time slots with length $\delta=T / K$. Based on the partial computation offloading model that we will discuss in the following, the time slot of interest is further divided into three stages, i.e., the laser WPT stage, the computation offloading stage, and the computing and downloading stage, as depicted in Fig. 2 We use $t^{\mathrm{W}}$ to represent the time of the laser WPT for each AUE. Similar to $[28|-| 30]$, the time consumed at the computing and downloading stage of the HAP are further neglected since the HAP has a much powerful computation capability (e.g., a high-speed multi-core CPU) and a much higher transmit power than the AUEs. Therefore, at each time slot, the HAP first uses a fraction $t^{\mathrm{W}} / \delta$ of the time slot to deliver laser-beamed energy concurrently for all the AUEs, and then each AUE completes the partial computation offloading during the remaining $1-\left(t^{\mathrm{W}} / \delta\right)$ fraction of time by using the harvested energy. 


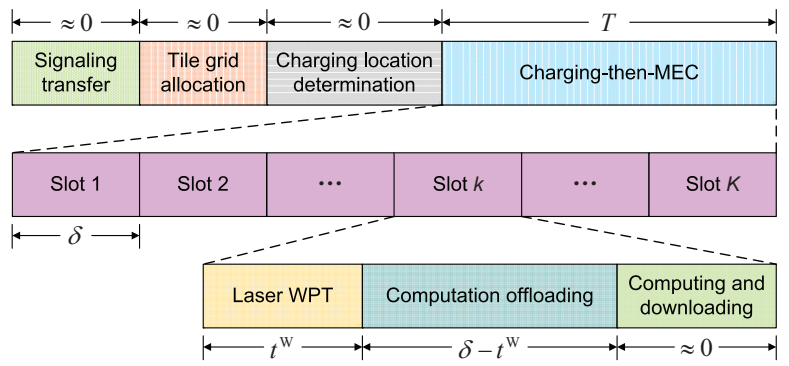

Fig. 2. Frame structure of the considered laser-powered aerial MEC system.

\subsection{Laser WPT Model}

With the time-switched "charging-then-MEC" strategy, the HAP wirelessly transfers the laser-beamed energy concurrently to all the AUEs at each time slot through laser beaming. We assume that the HAP adopts a fixed optical laser power $\beta$ of the associated laser beam at each time slot. With the LoS link established by the HAP, the received optical laser power for AUE $n$ in tier $\gamma$ at time slot $k$ can be given by [12], [31]

$$
E_{n}^{\mathrm{R}}[k]=t^{\mathrm{W}} \beta \frac{A}{\left(D+d_{n}^{(\gamma)} \Delta \theta\right)^{2}} \chi e^{-d_{n}^{(\gamma)} \zeta},
$$

where $A$ is the area of the receiver telescope or collection lens, $D$ is the size of the initial laser beam, $\Delta \theta$ is the angular spread, $\chi$ is the combined transmission receiver optical efficiency, and $\zeta$ is the attenuation coefficient of the medium (in $\mathrm{m}^{-1}$ ). By considering the conventional linear energy harvesting models, the actual harvested laser energy of AUE $n$ in tier $\gamma$ at time slot $k$ can be computed as a linear function of the input laser-beamed energy, i.e.,

$$
E_{n}^{\mathrm{H}}[k]=\tau E_{n}^{\mathrm{R}}[k]=\frac{\tau t^{\mathrm{W}} \beta A \chi e^{-d_{n}^{(\gamma)} \zeta}}{\left(D+d_{n}^{(\gamma)} \Delta \theta\right)^{2}},
$$

where $\tau \in(0,1)$ is the laser energy conversion efficiency. Accordingly, the actual harvested laser energy of AUE $n$ in tier $\gamma$ during time horizon $T$ can be obtained as

$$
E_{n}^{\mathrm{H}}=\sum_{k=1}^{K} E_{n}^{\mathrm{H}}[k]=\sum_{k=1}^{K} \tau E_{n}^{\mathrm{R}}[k]=\frac{\tau t^{\mathrm{W}} \beta A \chi e^{-d_{n}^{(\gamma)} \zeta}}{\left(D+d_{n}^{(\gamma)} \Delta \theta\right)^{2}} .
$$

\subsection{Communication Model}

To offload the computation task to the HAP via the A2A uplink transmission during the charging-then-MEC phase, it is assumed that each AUE is assigned to an orthogonal subchannel over the resource block (RB). Each subchannel has an equally-sized bandwidth, denoted by $W$. It is noticeable that the co-channel interference can be completely avoided for all the AUEs during the A2A uplink transmissions within the 3D coverage space $\Omega$.

We further suppose that the HAP operates in the Kaband sharing the same RB based on the ITU-R spectrum regulation $[8]$. By considering that the $\mathrm{A} 2 \mathrm{~A}$ transmission links between the AUEs and the HAP are dominated by the cleared LoS links, the channel fading thus depends on both the free space path loss and the miscellaneous atmospheric loss [26]. As such, the path loss between AUE $n$ in tier $\gamma$ and the HAP at time slot $k$ can be expressed by

$$
l_{n}[k]=\left(\frac{4 \pi \phi_{n} d_{n}^{(\gamma)}}{c}\right)^{\alpha}+l_{\mathrm{A}},
$$

where $\phi_{n}$ is the carrier frequency of subchannel for AUE $n$ in tier $\gamma, c$ is the speed of light, $\alpha \geq 2$ is the path loss exponent, and $l_{\mathrm{A}}$ is the atmospheric loss depending on the effect of oxygen and water vapour on the A2A transmission link. Note that the path loss can be referred to as the fixed value for the AUE at each time slot due to the fixed distance between the HAP and the AUE during time horizon $T$. Therefore, the channel gain between AUE $n$ in tier $\gamma$ and the HAP at time slot $k$ can be calculated as $g_{n}[k]=\frac{1}{l_{n}[k]}$. As a result, the achievable uplink rate of AUE $n$ in tier $\gamma$ to the $\mathrm{HAP}$ at time slot $k$ can be formulated as

$$
R_{n}^{\mathrm{U}}[k]=W \log _{2}\left(1+\frac{p_{n}[k] g_{n}[k]}{\sigma^{2}}\right),
$$

where $p_{n}[k]$ is the transmit power of AUE $n$ in tier $\gamma$ to the HAP at time slot $k$, and $\sigma^{2}$ is the variance of the AWGN at the HAP. As for the transmit power for the AUE in A2A uplink transmission, note that the distance between the HAP and the AUE is a fixed value during time horizon $T$, it does not make sense to discuss the power optimization. Therefore, we assume there is no power control in A2A uplink transmissions for the AUEs and their transmit power is fixed at each time slot, but may continuously vary over the different time slots.

\subsection{Computing Model}

In this paper, we adopt the partial computation offloading model for each AUE during the charging-then-MEC phase. With this model, the computation task of each AUE can either be executed locally by itself, or be offloaded via the A2A uplink transmission to and executed by the HAP. We employ the size of computation data including the program codes and the input parameters to characterize the computation task for each AUE. Without loss of generality, the computation task of AUE $n$ in tier $\gamma$ at time slot $k$ is defined by a two-tuple $w_{n}[k]=\left\{B_{n}[k], \xi_{n}[k]\right\}$, where $B_{n}[k]$ (in bits) is the total data size of computation task for AUE $n$, and $\xi_{n}[k]$ (in bits) is the data size of computation task offloaded from AUE $n$ to the HAP.

\subsubsection{Local Computing}

For local task computing, the data size of computation task executed by AUE $n$ at time slot $k$ is denoted as $B_{n}[k]-\xi_{n}[k]$ (in bits) for task $w_{n}[k]$. We assume that the local computing capability chosen by each AUE is fixed when computing during time horizon $T$, but may continuously vary over the different AUEs. Let $f_{n}$ represent the local computing capability (in CPU cycles/s) of AUE $n$. Given each time slot, we denote the number of CPU cycles required to process one bit of raw data at AUE $n$ by $\varpi_{n}$, which is determined by the nature of resource-hungry applications and is further assumed to be equal for each AUE. Therefore, 
the computing rate (in bps) for local computing at AUE $n$ at time slot $k$ is calculated by

$$
r_{n}^{\mathrm{L}}[k]=\frac{f_{n}}{\varpi_{n}} .
$$

Noticing that the rate $r_{n}^{\mathrm{L}}[k]$ at time slot $k$ is also equivalent to the local computing rate of AUE $n$ during time horizon $T$, i.e., $r_{n}^{\mathrm{L}}=r_{n}^{\mathrm{L}}[k], \forall k$. The computation delay for processing task $w_{n}[k]$ locally at AUE $n$ at time slot $k$ is then formulated as $t_{n}^{\mathrm{L}}[k]=\frac{B_{n}[k]-\xi_{n}[k]}{r_{n}^{L}[k]}$. Thereby, the total computation delay for local computing at AUE $n$ during time horizon $T$ can be expressed as

$$
T_{n}^{\mathrm{L}}=\sum_{k=1}^{K} t_{n}^{\mathrm{L}}[k]=\sum_{k=1}^{K} \frac{\left(B_{n}[k]-\xi_{n}[k]\right) \varpi_{n}}{f_{n}} .
$$

Based on the configuration of CPU architecture, the power consumption for local computing of AUE $n$ at time slot $k$ can be characterized by $q_{n}^{\mathrm{L}}[k]=\kappa f_{n}^{3}$, where $\kappa$ is an effective switched capacitance depending on the chip architecture of CPU for each AUE [17|. With that in mind, the energy consumption for local computing of AUE $n$ at time slot $k$ can be modeled as $E_{n}^{\mathrm{L}}[k]=\kappa f_{n}^{3} t_{n}^{\mathrm{L}}[k]$. Correspondingly, the total energy consumption for local computing of AUE $n$ during time horizon $T$ is then formulated by

$$
E_{n}^{\mathrm{L}}=\sum_{k=1}^{K} E_{n}^{\mathrm{L}}[k]=\sum_{k=1}^{K} \kappa f_{n}^{2} \varpi_{n}\left(B_{n}[k]-\xi_{n}[k]\right) .
$$

\subsubsection{Computation Offloading}

By incorporating the A2A uplink transmission overhead (e.g., encryption and packet header), denoted by $\vartheta_{n}$, the actual data size of computation task offloaded from AUE $n$ to the HAP at time slot $k$ is defined as $\vartheta_{n} \xi_{n}[k]$. Then the uplink transmission delay of AUE $n$ for offloading the task to the HAP at time slot $k$ can be obtained by $t_{n}^{\mathrm{U}}[k]=\frac{\vartheta_{n} \xi_{n}[k]}{R_{n}^{\mathrm{U}}[k]}$. As a result, the total uplink transmission delay for computation offloading at AUE $n$ during time horizon $T$ is expressed as

$$
T_{n}^{\mathrm{U}}=\sum_{k=1}^{K} t_{n}^{\mathrm{U}}[k]=\sum_{k=1}^{K} \frac{\vartheta_{n} \xi_{n}[k]}{R_{n}^{\mathrm{U}}[k]} .
$$

By considering the transmit power for offloading, the energy consumption for the A2A uplink transmission of AUE $n$ offloading the task to the HAP at time slot $k$ is written by $E_{n}^{\mathrm{U}}[k]=p_{n}[k] t_{n}^{\mathrm{U}}[k]$. Therefore, the total energy consumption for computation offloading at AUE $n$ during time horizon $T$ is given by

$$
E_{n}^{\mathrm{U}}=\sum_{k=1}^{K} E_{n}^{\mathrm{U}}[k]=\sum_{k=1}^{K} \frac{\vartheta_{n} \xi_{n}[k] p_{n}[k]}{R_{n}^{\mathrm{U}}[k]} .
$$

\subsection{Flight Energy Consumption Model}

The flight energy consumption for the AUEs in the fixedwing configuration mainly depends on their propulsion energy consumption to maintain the airborne as well as to support their velocities and accelerations. For the sake of simplicity, we assume that each AUE has the same flight energy consumption during the charging-then-MEC phase. Utilizing the results of [32], the flight energy consumed by AUE $n$ during time horizon $T$ can be analytically obtained by (12) as shown at the top of the next page, where $\ell_{1}$ and $\ell_{2}$ are parameters related to the weight, wing area, air density of AUE $n, m$ is the mass of AUE $n$ including all the payloads, $g$ is the acceleration of gravity, the superscript $\mathbf{a}_{n}^{\mathrm{T}}$ is the transpose of vector $\mathbf{a}_{n}$, and $\mathbf{v}_{n}[k]$ and $\mathbf{a}_{n}[k]$ are the velocity and acceleration vectors of AUE $n$ at time slot $k$, respectively, which are subject to the following constraints over the time slots, for $k \in\{1,2, \cdots, K-1\}$, i.e.,

$$
\begin{gathered}
\mathbf{v}_{n}[k+1]=\mathbf{v}_{n}[k]+\delta \cdot \mathbf{a}_{n}[k], \\
\mathbf{q}_{n}^{(\gamma)}[k+1]=\mathbf{q}_{n}^{(\gamma)}[k]+\delta \cdot \mathbf{v}_{n}[k]+\frac{1}{2} \delta^{2} \cdot \mathbf{a}_{n}[k] .
\end{gathered}
$$

\subsection{Adversary Model}

Due to the openness of wireless transmissions for the WPT signaling messages on the control channels, we thus take into account an external adversary who misbehaves or deviates from the legitimate laser charging operations required by the AUEs. The adversary is assumed to be deployed on the ground with a fixed location, as shown in Fig. 1. The goal of the adversary is to capture the wireless communications during the signaling transfer phase, and analyze the transmitted WPT signaling messages from the AUEs to track their locations and learn about the contents of the signaling messages, e.g, the preferences of charging locations for the AUEs. Based on the observation of the WPT signaling transfer, the adversary aims to actively launch the CLDF attacks by sending the false charging location data to the AUEs during the tile grid allocation phase. Particularly, we consider two types of attacks that can be mounted by the adversary:

- Eavesdropping. By applying a smart and programmable radio device such as one mentioned in [33], the adversary listens on the control channels during the signaling transfer phase to eavesdrop on the WPT signaling messages transmitted from the AUEs and read the contents of the signaling messages. We assume that the adversary has the capability to perfectly detect when and where a WPT signaling message arrived from, that it has the same communication range as the AUEs in the system, and also has a large amount of memory to keep track of the signaling messages that have been overheard.

- Falsification. With the captured WPT signaling messages, the adversary can transmit a false charging location data in terms of tile grid to each AUE during the tile grid allocation phase, causing the AUE to receive the wrong $3 \mathrm{D}$ location of the charging point, which seriously violates the location privacy of the AUE. The CLDF attacks apparently suggest a compelling need for protecting the location privacy of the laser WPT in the laser-powered aerial MEC system.

Note that here we don't consider the adversary model that can modify or falsify the offloaded computation task, 


$$
E_{n}^{\mathrm{F}}=\sum_{k=1}^{K} \delta\left[\ell_{1}\left\|\mathbf{v}_{n}[k]\right\|^{3}+\frac{\ell_{2}}{\left\|\mathbf{v}_{n}[k]\right\|}\left(1+\frac{\left\|\mathbf{a}_{n}[k]\right\|^{2}-\frac{\left(\mathbf{a}_{n}^{\mathrm{T}}[k] \mathbf{v}_{n}[k]\right)^{2}}{\left\|\mathbf{v}_{n}[k]\right\|^{2}}}{g^{2}}\right)\right]+\frac{1}{2} m\left(\left\|\mathbf{v}_{n}[K]\right\|^{2}-\left\|\mathbf{v}_{n}[1]\right\|^{2}\right),
$$

because our proposed tile grid allocation game framework we will describe in Section 4 is based on the assumption that all the offloaded data are correct during the chargingthen-MEC phase.

In this paper, we only focus on the CLDF attacks mounted by the adversary through distributing the false tile grids over the AUEs to compromise the location privacy for laser charging. While the adversary tries to allocate its attack resources regarding the false tile grids over the AUEs, the HAP will act as a defender that seeks to optimally allocate its defense resources in terms of the legitimate tile grids to prevent the adversary from causing the unsuccessful laser WPT and the failure of the aerial MEC. Such an attackdefense resource allocation during the tile grid allocation phase allows us to gain more insights into the competitive interactions between the defender and the adversary in their simultaneous distributions for the fixed number of tile grids across the AUEs. To formulate the competitive resource allocation problem under such an attack-defense scenario, in Section 4. we will employ the Colonel Blotto game framework [34]-|36] for strategically distributing tile grids over the AUEs for both the defender and the adversary.

\section{Game Formulation and Solution}

In this section, we propose the Colonel Blotto game framework in Subsection 4.1 to formulate the competitive interactions between the defender and the adversary for simultaneously allocating their tile grids, respectively, across a finite number of AUEs, and then describe the solution to the proposed tile grid allocation game with symmetric players in Subsection 4.2 and asymmetric players in 4.3 respectively.

\subsection{Colonel Blotto Game Formulation}

The Colonel Blotto game [34], [35], [37] is one popular game-theoretic framework for modeling and analyzing the strategic resource allocation of two players in a competitive environment, to simultaneously distribute limited resources over a given number of battlefields. Therefore, the interactions between the defender and the adversary regarding their competitive allocations of the fixed number of tile grids among $N$ AUEs can be formulated as a Colonel Blotto game with $N$ battlefields. In this game, the defender chooses the defense tile grid distribution vector over $N$ battlefields during the tile grid allocation phase, while the adversary chooses the attack tile grid distribution vector. The player that allocates a higher amount of tile grids to the individual battlefield wins in that battlefield. Formally, the Colonel Blotto game framework is defined in the following.

Definition 1 (Pure Strategy): A Colonel Blotto game with a pure strategy for tile grid allocation of $N$ AUEs during the tile grid allocation phase is formulated as a 4-tuple:

$$
\mathfrak{C B}_{1}=\left\{\mathcal{P}, \mathcal{N},\left\{\mathscr{X}_{n}^{[T]}\right\}_{n \in \mathcal{N}},\left\{u_{\mathscr{X}, n}^{[T]}\right\}_{n \in \mathcal{N}}\right\},
$$

- Player set $\mathcal{P}: \mathcal{P}=\{H, S\}$, wherein there are two players, player $H$ (referred to as the defender) and player $S$ (referred to as the adversary), that compete by simultaneously distributing the tile grids over $N$ AUEs.

- Battlefield set $\mathcal{N}$ : A battlefield stands for an AUE. There are $N$ homogeneous battlefields, denoted by a set $\mathcal{N}$.

- Action space $\left\{\mathscr{X}_{n}^{[T]}\right\}_{n \in \mathcal{N}}$ : Each player allocates the number of tile grids $\mathscr{X}_{n}^{[T]}$ to battlefield $n$ during the tile grid allocation phase, for $\mathscr{X} \triangleq H$ (defender) and $\mathscr{X} \triangleq S$ (adversary). The tile grid distribution vectors of the defender and the adversary across battlefield set $\mathcal{N}$ are represented by $\mathbf{H}[T]=\left\{H_{n}^{[T]}\right\}_{n \in \mathcal{N}}$ and $\mathbf{S}[T]=\left\{S_{n}^{[T]}\right\}_{n \in \mathcal{N}}$, respectively. The total number of tile grids distributed by the defender and the adversary across battlefield set $\mathcal{N}$ during the tile grid allocation phase are given as $Z_{H}^{[T]}$ and $Z_{S}^{[T]}$, for $Z_{H}^{[T]}, Z_{S}^{[T]} \leq Q \times Q \times Y$, respectively. Let $\mathbb{Z}_{+}$denote the set of all non-negative integers. Therefore, the action sets of the feasible allocations of tile grids for the defender and the adversary over $N$ battlefields are respectively defined by

$$
\begin{gathered}
\boldsymbol{\Gamma}_{H}=\left\{\left\{H_{n}^{[T]}\right\}_{n \in \mathcal{N}} \subset \mathbb{Z}_{+} \mid Z_{H}^{[T]}=\sum_{n=1}^{N} H_{n}^{[T]}\right\}, \\
\boldsymbol{\Gamma}_{S}=\left\{\left\{S_{n}^{[T]}\right\}_{n \in \mathcal{N}} \subset \mathbb{Z}_{+} \mid Z_{S}^{[T]}=\sum_{n=1}^{N} S_{n}^{[T]}\right\} .
\end{gathered}
$$

- Utility function $\left\{u_{\mathscr{X}, n}^{[T]}\right\}_{n \in \mathcal{N}}$ : The utility obtained by each player from battlefield $n$ during the chargingthen-MEC phase within time horizon $T$ is expressed as $u_{\mathscr{X}, n}^{[T]}$. The total utilities of the defender and the adversary are given by $u_{H}^{[T]}=\sum_{n=1}^{N} u_{H, n}^{[T]}$ and $u_{S}^{[T]}=\sum_{n=1}^{N} u_{S, n}^{[T]}$, respectively. Considering that the Colonel Blotto game is a type of zero-sum game, the utilities of two players always add up to zero, i.e., $u_{H, n}^{[T]}=-u_{S, n}^{[T]}$ and $u_{H}^{[T]}=-u_{S}^{[T]}$.

For the tile grid allocation game $\mathfrak{C B}_{1}$ with pure strategy, the defender and the adversary compete by simultaneously announcing distributions of the number of tile grids $H_{n}^{[T]}$ and $S_{n}^{[T]}$ on battlefield $n$, respectively. Typically, each battlefield is won by the player that allocates a larger number of tile grids on that battlefield. To this end, the main idea behind the proposed privacy-aware laser WPT in this paper is to compare the number of distributed tile grids for each battlefield between two players. Then, we can infer that when $H_{n}^{[T]}>S_{n}^{[T]}$, the defender wins battlefield $n$. Otherwise, when $H_{n}^{[T]} \leq S_{n}^{[T]}$, the adversary wins battlefield $n$. From the above observations, we note that the player who 
wins more battlefields than its opponent finally wins this game. Hence, our objective in this game is to maximize the number of times that the defender wins across battlefield set $\mathcal{N}$ to prevent the adversary from launching the CLDF attacks.

To determine the utility function of each player obtained from the individual battlefield, we here take into account the impact of the charging-then-MEC phase on the competitive allocations of tile grids between the defender and the adversary during time horizon $T$. Matter of fact, the total energy consumption for local computing of AUE $n$ during time horizon $T$ should be less than $E_{n}^{\mathrm{H}}-E_{n}^{\mathrm{F}}-E_{n}^{\mathrm{U}}$. Considering the power consumption for local computing of AUE $n$ at time slot $k$, the maximum time used for local computing of AUE $n$ during time horizon $T$ can be calculated as $\frac{E_{n}^{\mathrm{H}}-E_{n}^{\mathrm{F}}-E_{n}^{\mathrm{U}}}{q_{n}^{\mathrm{L}}}$, where $q_{n}^{\mathrm{L}}=q_{n}^{\mathrm{L}}[k], \forall k$, due to the fixed local computing capability chosen by each AUE at each time slot. By combining the computing rate $r_{n}^{\mathrm{L}}$, the maximum processing input-bits of AUE $n$ during time horizon $T$ is interpreted by $\frac{r_{n}^{\mathrm{L}}\left(E_{n}^{\mathrm{H}}-E_{n}^{\mathrm{F}}-E_{n}^{\mathrm{U}}\right)}{q_{n}^{\mathrm{L}}}$. Let $B_{n}=\sum_{k=1}^{K} B_{n}[k]$ denote the total data size of computation task for AUE $n$ during time horizon $T$. Therefore, the local computation capacity for AUE $n$ during time horizon $T$ can be defined as follows

$$
R_{n}=\frac{r_{n}^{\mathrm{L}}\left(E_{n}^{\mathrm{H}}-E_{n}^{\mathrm{F}}-E_{n}^{\mathrm{U}}\right)}{q_{n}^{\mathrm{L}} B_{n}} .
$$

From the defender perspective, the local computation capacity for AUE $n$ during time horizon $T$ is rewritten as

$$
\widehat{R}_{n}= \begin{cases}\frac{r_{n}^{\mathrm{L}}\left(E_{n}^{\mathrm{H}}-E_{n}^{\mathrm{F}}-E_{n}^{\mathrm{U}}\right)}{q_{n}^{\mathrm{L}} B_{n}}, & H_{n}^{[T]}>S_{n}^{[T]}, \\ 0, & H_{n}^{[T]} \leq S_{n}^{[T]} .\end{cases}
$$

Since different AUEs may have different energy states and different requirements for the quality of computation service, both the energy consumption and execution delay are critical to the AUEs during the charging-then-MEC phase. To this aim, the utility function of each player from a given battlefield should be well developed by balancing the tradeoff among the local computation capacity, the energy consumption, and the execution delay of each AUE in the laser-powered aerial MEC system. Let $\lambda_{1}, \lambda_{2}$, and $\lambda_{3}$ represent the positive weighting factors used to ensure the same range for local computation capacity, energy consumption, and execution delay, respectively. With the considerations mentioned above, the utility that the defender receives on battlefield $n$ during time horizon $T$ in $\mathfrak{C B}_{1}$ with pure strategy can be determined by (20) as shown at the top of the next page.

To simplify the presentation, let us employ $\epsilon_{n}^{1}$ to stand for the utility that the defender receives on battlefield $n$ when $H_{n}^{[T]}>S_{n}^{[T]}$. Meanwhile, we further define $\epsilon_{n}^{2}$ as the utility that the defender receives on battlefield $n$ when $H_{n}^{[T]} \leq S_{n}^{[T]}$. For clarity of exposition, we then introduce a binary variable to represent the association relationship between $H_{n}^{[T]}$ and $S_{n}^{[T]}$ during time horizon $T$, i.e.,

$$
F\left(H_{n}^{[T]}, S_{n}^{[T]}\right)= \begin{cases}1, & H_{n}^{[T]}>S_{n}^{[T]} \\ 0, & H_{n}^{[T]} \leq S_{n}^{[T]}\end{cases}
$$

By combining (20) and (21), the total utilities obtained by the defender over battlefield set $\mathcal{N}$ during time horizon $T$ in $\mathfrak{C B}_{1}$ with pure strategy is thus written as

$$
\begin{aligned}
& u_{H}^{[T]}(\mathbf{H}, \mathbf{S})=-u_{S}^{[T]}(\mathbf{H}, \mathbf{S}) \\
= & \sum_{n=1}^{N}\left\{\epsilon_{n}^{1} F\left(H_{n}^{[T]}, S_{n}^{[T]}\right)+\epsilon_{n}^{2}\left(1-F\left(H_{n}^{[T]}, S_{n}^{[T]}\right)\right)\right\} .
\end{aligned}
$$

However, it has been revealed that the pure-strategy Nash equilibrium (NE) for the tile grid allocation game $\mathfrak{C} \mathfrak{B}_{1}$ does not always exist [34]. Indeed, given any pure strategies, the adversary can always modify its tile grid allocation strategy accordingly to improve its utility and win the game. Alternatively, for solving the proposed Colonel Blotto game to strategically distribute tile grids over battlefield set $\mathcal{N}$, a more general method is to find the mixed-strategy NE solution. In the case of mixed strategies, each player selects a probability distribution over the action space to maximize the potential expected total utilities.

Definition 2 (Mixed Strategy): A Colonel Blotto game with a mixed strategy for tile grid allocation of $N$ AUEs during the tile grid allocation phase is formulated as a 4-tuple:

$$
\mathfrak{C} \mathfrak{B}_{2}=\left\{\mathcal{P}, \mathcal{N},\left\{\mathscr{X}_{n, r}^{[T]}\right\}_{n=1, r=0}^{N, Z_{\mathscr{X}}^{[T]}},\left\{U_{\mathscr{X}}^{[T]}(\mathbf{h}, \mathbf{s})\right\}\right\}
$$

- Action space $\left\{\mathscr{X}_{n, r}^{[T]}\right\}_{n=1, r=0}^{N, Z_{\mathscr{X}}^{[T]}}$ : The probability that a player allocates $r$ tile grids to battlefield $n$ during the tile grid allocation phase is defined by $\mathscr{X}_{n, r}^{[T]}=$ $\operatorname{Pr}\left(\mathscr{X}_{n}^{[T]}=r\right)$, for $\mathscr{X} \triangleq H$ (defender) and $\mathscr{X} \triangleq$ $S$ (adversary). The probability distribution vectors of the defender and the adversary are denoted as $\mathbf{h}[T]=\left\{H_{n, r}^{[T]}\right\}_{n=1, r=0}^{N, Z_{H}^{[T]}}$ and $\mathbf{s}[T]=\left\{S_{n, r}^{[T]}\right\}_{n=1, r=0}^{N, Z_{S}^{[T]}}$ respectively.

- Utility function $\left\{U_{\mathscr{X}}^{[T]}(\mathbf{h}, \mathbf{s})\right\}$ : The expected total utilities obtained by each player over battlefield set $\mathcal{N}$ during the charging-then-MEC phase within time horizon $T$ is described as $U_{\mathscr{X}}^{[T]}(\mathbf{h}, \mathbf{s})$. Due to the fact that the Colonel Blotto game is a type of zero-sum game, the expected total utilities of two players always add up to zero, which can be further expressed as 24) as shown at the top of the next page.

Based on $\mathfrak{C B}_{2}$ with mixed strategy, we define $\psi_{i, r}^{[T]}$ and $\varphi_{i, r}^{[T]}$ by the $i$-th highest probability of $H_{n, r}^{[T]}$ and the $i$-th highest probability of $S_{n, r}^{[T]}$, respectively, for $i \in \mathcal{N}$. Then the mixed-strategy action sets for the defender and the adversary can be respectively determined by

$$
\begin{gathered}
\boldsymbol{\Pi}_{H}=\left\{\left\{\psi_{i, r}^{[T]}\right\}_{i=1, r=0}^{N, Z_{H}^{[T]}} \mid \psi_{i, r}^{[T]} \geq H_{n, r}^{[T]}, \sum_{r=0}^{Z_{H}^{[T]}} \psi_{i, r}^{[T]}=1\right\}, \\
\boldsymbol{\Pi}_{S}=\left\{\left\{\varphi_{i, r}^{[T]}\right\}_{i=1, r=0}^{N, Z_{S}^{[T]}} \mid \varphi_{i, r}^{[T]} \geq S_{n, r}^{[T]}, \sum_{r=0}^{Z_{S}^{[T]}} \varphi_{i, r}^{[T]}=1\right\} .
\end{gathered}
$$




$$
u_{H, n}^{[T]}(\mathbf{H}, \mathbf{S})=-u_{S, n}^{[T]}(\mathbf{H}, \mathbf{S})= \begin{cases}\lambda_{1} \frac{r_{n}^{\mathrm{L}}\left(E_{n}^{\mathrm{H}}-E_{n}^{\mathrm{F}}-E_{n}^{\mathrm{U}}\right)}{q_{n}^{\mathrm{L}} B_{n}}-\lambda_{2}\left(E_{n}^{\mathrm{L}}+E_{n}^{\mathrm{U}}\right)-\lambda_{3}\left(T_{n}^{\mathrm{L}}+T_{n}^{\mathrm{U}}\right), & H_{n}^{[T]}>S_{n}^{[T]}, \\ 0, & H_{n}^{[T]} \leq S_{n}^{[T]} .\end{cases}
$$

$$
U_{H}^{[T]}(\mathbf{h}, \mathbf{s})=-U_{S}^{[T]}(\mathbf{h}, \mathbf{s})=\underset{\substack{\mathbf{s} \sim \mathbf{S} \\ \mathbf{h} \sim \mathbf{H}}}{\mathbb{E}}\left[\sum_{n=1}^{N}\left\{\epsilon_{n}^{1} F\left(H_{n}^{[T]}, S_{n}^{[T]}\right)+\epsilon_{n}^{2}\left(1-F\left(H_{n}^{[T]}, S_{n}^{[T]}\right)\right)\right\}\right]
$$

\subsection{Mixed Strategy NE Solution under Symmetric Play- ers}

In this subsection, we begin by finding the mixed-strategy $\mathrm{NE}$ solution to the tile grid allocation game $\mathfrak{C B}_{2}$, which is defined as follows.

Definition 3 (Nash Equilibrium): A tile grid distribution profile $\left(\mathbf{h}^{*}, \mathbf{s}^{*}\right)$ is a mixed-strategy NE point of $\mathfrak{C B}_{2}$ with mixed strategy if and only if no player can improve its utility by deviating unilaterally, i.e.,

$$
\begin{aligned}
& U_{H}^{[T]}\left(\mathbf{h}^{*}, \mathbf{s}^{*}\right) \geq U_{H}^{[T]}\left(\mathbf{h}, \mathbf{s}^{*}\right), \forall \mathbf{h} \in \mathbf{\Pi}_{H}, \\
& U_{S}^{[T]}\left(\mathbf{h}^{*}, \mathbf{s}^{*}\right) \geq U_{S}^{[T]}\left(\mathbf{h}^{*}, \mathbf{s}\right), \quad \forall \mathbf{s} \in \mathbf{\Pi}_{S} .
\end{aligned}
$$

For ease of exposition, let us first consider the tile grid allocation game $\mathfrak{C B}_{2}$ with symmetric players, in which two players have the same amount of resources (i.e., the same number of tile grids) to distribute across the battlefield set $\mathcal{N}$. This implies that the total number of tile grids distributed by the defender and the adversary are completely equal, i.e., $Z_{H}^{[T]}=Z_{S}^{[T]}$. In this way, the defender and the adversary can uniformly deploy the tile grids over battlefield set $\mathcal{N}$, respectively. Due to the non-negative integer constraints imposed on the tile grid allocation, the mixedstrategy Colonel Blotto game is actually referred to as a constant-sum two-player matrix game with a finite number of strategies [38]. We then use $\mathbf{1}_{h \times s}$ and $\mathbf{0}_{h \times s}$ to denote the all-one and all-zero $h \times s$ matrices, respectively. Let $\lfloor\cdot\rfloor$ refer to the lower floor function. More specifically, the mixedstrategy NE solution to $\mathfrak{C B}_{2}$ with symmetric resources is analytically derived as the following theorem.

Theorem 1: If $Z_{H}^{[T]}=Z_{S}^{[T]}$ and $B_{n} \leq \sum_{m=1, m \neq n}^{N} B_{m}, a$ tile grid distribution profile $\left(\mathbf{h}^{*}, \mathbf{s}^{*}\right)$ constitutes a mixed-strategy $N E$ to the tile grid allocation game $\mathfrak{C B}_{2}$ with symmetric resources, where

$$
\mathbf{h}^{*}=\mathbf{s}^{*}=\left[\begin{array}{cc}
\frac{1}{\left\lfloor 2 \Upsilon B_{1}\right\rfloor+1} \mathbf{1}_{1 \times\left(\left\lfloor 2 \Upsilon B_{1}\right\rfloor+1\right)} & \mathbf{0}_{1 \times\left(Z_{H}^{[T]}-\left\lfloor 2 \Upsilon B_{1}\right\rfloor\right)} \\
\frac{1}{\left\lfloor 2 \Upsilon B_{2}\right\rfloor+1} \mathbf{1}_{1 \times\left(\left\lfloor 2 \Upsilon B_{2}\right\rfloor+1\right)} & \mathbf{0}_{1 \times\left(Z_{H}^{[T]}-\left\lfloor 2 \Upsilon B_{2}\right\rfloor\right)} \\
\vdots & \vdots \\
\frac{1}{\left\lfloor 2 \Upsilon B_{N}\right\rfloor+1} \mathbf{1}_{1 \times\left(\left\lfloor 2 \Upsilon B_{N}\right\rfloor+1\right)} & \mathbf{0}_{1 \times\left(Z_{H}^{[T]}\left\lfloor\left\lfloor\Upsilon B_{N}\right\rfloor\right)\right.}
\end{array}\right],
$$

where $\Upsilon=Z_{H}^{[T]} / \widehat{B}$.

Proof: Please see Appendix A

Corollary 1: With the mixed-strategy NE $\left(\mathbf{h}^{*}, \mathbf{s}^{*}\right)$ of the tile grid allocation game $\mathfrak{C B}_{2}$ with symmetric structure, the expected total utilities of two players are all equal to zero, i.e.,

$$
U_{H}^{[T]}\left(\mathbf{h}^{*}, \mathbf{s}^{*}\right)=-U_{S}^{[T]}\left(\mathbf{h}^{*}, \mathbf{s}^{*}\right)=0 .
$$

Proof: With the mixed-strategy NE solution under symmetric players, the number of tile grids assigned to each battlefield by the defender is equal to that of the adversary, i.e., the same uniform distribution of tile grids for two players. Referring to (36) and (37) in Appendix A, it can be obtained that no player can receive better utility than the other one. Intuitively, each battlefield will obtain the same probability of tile grid allocation from either the defender or the adversary. Based on (21), (24), and (29), we are able to directly derive the expected total utilities of two players as follows

$$
\begin{aligned}
& U_{H}^{[T]}\left(\mathbf{h}^{*}, \mathbf{s}^{*}\right)=-U_{S}^{[T]}\left(\mathbf{h}^{*}, \mathbf{s}^{*}\right) \\
&=\mathbb{E}_{\left(\mathbf{h}^{*}, \mathbf{s}^{*}\right)}\left[\sum_{n=1}^{N}\left\{\epsilon_{n}^{1} F\left(H_{n}^{[T]}, S_{n}^{[T]}\right)+\epsilon_{n}^{2}\left[1-F\left(H_{n}^{[T]}, S_{n}^{[T]}\right)\right]\right\}\right] \\
&=\mathbb{E}_{\left(\mathbf{h}^{*}, \mathbf{s}^{*}\right)}\left[\sum _ { n = 1 } ^ { N } \left\{\epsilon_{n}^{1} F\left(H_{n}^{[T]}, S_{n}^{[T]}\right) \mid F\left(H_{n}^{[T]}, S_{n}^{[T]}\right)=0,1\right.\right. \\
&\left.\left.+\epsilon_{n}^{2}\left[1-F\left(H_{n}^{[T]}, S_{n}^{[T]}\right)\right] \mid F\left(H_{n}^{[T]}, S_{n}^{[T]}\right)=0,1\right\}\right] \\
&=\mathbb{E}_{\left(\mathbf{h}^{*}, \mathbf{s}^{*}\right)}\left[\sum _ { n = 1 } ^ { N } \left\{\left(\epsilon_{n}^{1} \times 0+\epsilon_{n}^{2} \times 1\right) \mid F\left(H_{n}^{[T]}, S_{n}^{[T]}\right)=0\right.\right. \\
&\left.\left.\quad+\left(\epsilon_{n}^{1} \times 1+\epsilon_{n}^{2} \times 0\right) \mid F\left(H_{n}^{[T]}, S_{n}^{[T]}\right)=1\right\}\right] \\
&=\mathbb{E}_{\left(\mathbf{h}^{*}, \mathbf{s}^{*}\right)}\left[\sum_{n=1}^{N} \epsilon_{n}^{1} \mid F\left(H_{n}^{[T]}, S_{n}^{[T]}\right)=1\right] \\
&=\sum_{n=1}^{N} \epsilon_{n}^{1}\left[1 \times \operatorname{Pr}\left(H_{n}^{*}>S_{n}^{*}\right)+0 \times \operatorname{Pr}\left(H_{n}^{*} \leq S_{n}^{*}\right)\right] \\
&=\sum_{n=1}^{N} \epsilon_{n}^{1}\left[\operatorname{Pr}\left(H_{n}^{*}>S_{n}^{*}\right) \mid H_{n}^{*}=S_{n}^{*}=r\right] \\
&=0 .
\end{aligned}
$$

This completes the proof.

Remark 1: Under the condition of the same amount of resources to distribute for tile grid allocation game $\mathfrak{C B}_{2}$ with symmetric players, the defender and the adversary both choose the number of tile grids to uniformly allocate for each AUE within the interval $\left.\left[0, \mid 2 Z_{H}^{[T]} B_{n} / \widehat{B}\right\rfloor\right]$, and no battlefield dominates in the game, i.e., $B_{n} \leq$ $\sum_{m=1, m \neq n}^{N} B_{m}$. Moreover, the defender and the adversary 
then have the same probability uniform distribution during the tile grid allocation phase. From this result, we observe that no player wins the game, and no AUE can receive the spatial location to charge via the laser WPT. Therefore, tile grid allocation game $\mathfrak{C B}_{2}$ with symmetric players yields zero utility of the defender.

\subsection{Mixed Strategy NE Solution under Asymmetric Players}

In contrast with the symmetric structure, herein we consider the tile grid allocation game $\mathfrak{C} \mathfrak{B}_{2}$ with asymmetric players. In this case, the defender and the adversary have different numbers of tile grids, i.e., $Z_{H}^{[T]}>Z_{S}^{[T]}$, to deploy over the battlefield set $\mathcal{N}$. Formally, the mixed-strategy NE solution of $\mathfrak{C B}_{2}$ with asymmetric resources is characterized as follows.

Theorem 2: If $2 / N \leq Z_{S}^{[T]} / Z_{H}^{[T]} \leq 1$ and $N \geq 3$, a tile grid distribution profile $\left(\mathbf{h}^{*}, \mathbf{s}^{*}\right)$ constitutes a mixed-strategy $N E$ to the tile grid allocation game $\mathfrak{C B}_{2}$ with asymmetric resources, given by (32) and (33) shown at the top of next page, respectively, where $\Xi=Z_{H}^{[T]} / N$.

Proof: Please see Appendix B.

Corollary 2: With the mixed-strategy $N E\left(\mathbf{h}^{*}, \mathbf{s}^{*}\right)$ to the tile grid allocation game $\mathfrak{C B}_{2}$ with asymmetric structure, the expected total utilities of two players are achieved as

$$
\begin{aligned}
U_{H}^{[T]}\left(\mathbf{h}^{*}, \mathbf{s}^{*}\right) & =-U_{S}^{[T]}\left(\mathbf{h}^{*}, \mathbf{s}^{*}\right) \\
& =\left(1-\frac{Z_{S}^{[T]}}{Z_{H}^{[T]}}\right) \sum_{n=1}^{N} \epsilon_{n}^{1} .
\end{aligned}
$$

Proof: As observed from the mixed-strategy NE to $\mathfrak{C B}_{2}$, the number of tile grids assigned to each battlefield by the defender is different from that of the adversary, i.e., $H_{n}^{*}>S_{n}^{*}$ and $H_{n}^{*} \leq S_{n}^{*}$. Moreover, the probability of such the different cases can be denoted by $\operatorname{Pr}\left(H_{n}^{*}>S_{n}^{*}\right)$ and $\operatorname{Pr}\left(H_{n}^{*} \leq S_{n}^{*}\right)$, respectively. Referring to (19) and (21), it can be readily seen that the defender can obtain the expected total utilities, i.e., $\operatorname{Pr}\left(H_{n}^{*}>S_{n}^{*}\right)$, if and only if $H_{n}^{*}>S_{n}^{*}$. Based on (21), (24), 32), and (33), we thus derive the expected total utilities of two players as follows

$$
\begin{gathered}
U_{H}^{[T]}\left(\mathbf{h}^{*}, \mathbf{s}^{*}\right)=-U_{S}^{[T]}\left(\mathbf{h}^{*}, \mathbf{s}^{*}\right) \\
=\mathbb{E}_{\left(\mathbf{h}^{*}, \mathbf{s}^{*}\right)}\left[\sum_{n=1}^{N}\left\{\epsilon_{n}^{1} F\left(H_{n}^{[T]}, S_{n}^{[T]}\right)+\epsilon_{n}^{2}\left[1-F\left(H_{n}^{[T]}, S_{n}^{[T]}\right)\right]\right\}\right] \\
=\mathbb{E}_{\left(\mathbf{h}^{*}, \mathbf{s}^{*}\right)}\left[\sum _ { n = 1 } ^ { N } \left\{\epsilon_{n}^{1} F\left(H_{n}^{[T]}, S_{n}^{[T]}\right) \mid F\left(H_{n}^{[T]}, S_{n}^{[T]}\right)=0,1\right.\right. \\
\left.\left.+\epsilon_{n}^{2}\left[1-F\left(H_{n}^{[T]}, S_{n}^{[T]}\right)\right] \mid F\left(H_{n}^{[T]}, S_{n}^{[T]}\right)=0,1\right\}\right] \\
=\mathbb{E}_{\left(\mathbf{h}^{*}, \mathbf{s}^{*}\right)}\left[\sum _ { n = 1 } ^ { N } \left\{\left(\epsilon_{n}^{1} \times 0+\epsilon_{n}^{2} \times 1\right) \mid F\left(H_{n}^{[T]}, S_{n}^{[T]}\right)=0\right.\right. \\
\left.\left.+\left(\epsilon_{n}^{1} \times 1+\epsilon_{n}^{2} \times 0\right) \mid F\left(H_{n}^{[T]}, S_{n}^{[T]}\right)=1\right\}\right]
\end{gathered}
$$

$$
\begin{aligned}
& =\mathbb{E}_{\left(\mathbf{h}^{*}, \mathbf{s}^{*}\right)}\left[\sum_{n=1}^{N} \epsilon_{n}^{1} \mid F\left(H_{n}^{[T]}, S_{n}^{[T]}\right)=1\right] \\
& =\sum_{n=1}^{N} \epsilon_{n}^{1}\left[1 \times \operatorname{Pr}\left(H_{n}^{*}>S_{n}^{*}\right)+0 \times \operatorname{Pr}\left(H_{n}^{*} \leq S_{n}^{*}\right)\right] \\
& =\sum_{n=1}^{N} \epsilon_{n}^{1}\left[\operatorname{Pr}\left(H_{n}^{*}>S_{n}^{*}\right) \mid S_{n}^{*}=0, S_{n}^{*} \neq 0\right] \\
& =\sum_{n=1}^{N} \epsilon_{n}^{1}\left[\operatorname{Pr}\left(H_{n}^{*}>0\right)+\operatorname{Pr}\left(H_{n}^{*}>S_{n}^{*}\right) \mid H_{n}^{*}=S_{n}^{*}=r, S_{n}^{*} \neq 0\right] \\
& =\left(1-\frac{Z_{S}^{[T]}}{Z_{H}^{[T]}}\right) \sum_{n=1}^{N} \epsilon_{n}^{1} .
\end{aligned}
$$

As a result, we obtain the desired expression as (34). The proof is complete.

Remark 2: Our objective in this work is to achieve the improvement of the total utilities of the defender by effectively identifying a tradeoff among the local computation capacity, the energy consumption, and the execution delay for each AUE in the laser-powered aerial MEC system. The defender has to distribute more tile grids than the adversary to protect the location privacy for laser charging of the AUEs. When $Z_{H}^{[T]}>Z_{S}^{[T]}$, the defender guarantees that more AUEs will obtain the laser WPT, which ensures that more computation tasks can be completed locally or offloading to the HAP. In other words, the defender wins the game and the total utilities increase accordingly with the growing number of tile grids allocated by the defender.

\section{Simulation Results}

In this section, we provide the results of numerical simulations to illustrate the theoretical analyses and validate the performance of the proposed framework via the Colonel Blotto game with different parameter settings. Since no other existing work studies the same problem, we here compare the proposed framework against the design baselines by defining a privacy protection level, denoted as $\Phi \in(0,1)$. Note that this paradigm reveals the proportion of the number of the AUEs with the enhanced location privacy for laser WPT with the help of our proposed framework.

Throughout the simulations, all the results are obtained with the following default system settings. We consider the laser-powered aerial MEC system consisting of an HAP deployed in a quasi-stationary position at the stratospheric altitude of $L_{\mathrm{H}}=21 \mathrm{~km}$ and an adversary deployed on the ground with a fixed location. All the AUEs are intelligently flying with the random flight trajectories within the considered coverage space $\Omega$ of the HAP. During the charging-then$M E C$ phase, the time horizon is assumed to be $T=2 \mathrm{~s}$ which is equally divided into $K=5$ time slots. For each time slot, the HAP applies half of the time slot to deliver the laserbeamed energy towards all the AUEs, for $t^{\mathrm{W}}=\frac{\delta}{2}$. We set the horizontal coordinate of the HAP as $\mathbf{q}^{\mathrm{H}}=(10.5 \mathrm{~m}, 40.5 \mathrm{~m})$, and choose the horizontal coordinate of each AUE according to the interval $[(10.5 \mathrm{~m}, 11.5 \mathrm{~m}),(29.5 \mathrm{~m}, 60.5 \mathrm{~m})]$ during 


$$
\begin{gathered}
\mathbf{h}^{*}=\left[\begin{array}{lll}
\mathbf{0}_{N \times 1} & \frac{1}{\lfloor 2 \Xi\rfloor} \mathbf{1}_{N \times\lfloor 2 \Xi\rfloor} & \mathbf{0}_{N \times\left(Z_{H}^{[T]}-\lfloor 2 \Xi\rfloor\right)}
\end{array}\right], \\
\mathbf{s}^{*}=\left[\begin{array}{lll}
\left(1-\frac{Z_{S}^{[T]}}{Z_{H}^{[T]}}\right) \mathbf{1}_{N \times 1} & \frac{Z_{S}^{[T]}}{Z_{H}^{[T]}\lfloor 2 \Xi\rfloor} \mathbf{1}_{N \times\lfloor 2 \Xi\rfloor} & \mathbf{0}_{N \times\left(Z_{H}^{[T]}-\lfloor 2 \Xi\rfloor\right)}
\end{array}\right],
\end{gathered}
$$

TABLE 1

Simulation Parameters.

\begin{tabular}{|l|l|l|}
\hline Parameter & Description & Value \\
\hline \hline$\tau$ & Energy conversion efficiency & 0.7 \\
\hline$A$ & Area of the receiver telescope & $7.85 \times 10^{-3} \mathrm{~m}^{2}$ \\
\hline$D$ & Size of the initial laser beam & $0.1 \mathrm{~m}$ \\
\hline$\Delta \theta$ & Angular spread & $3.4 \times 10^{-8}$ \\
\hline$\chi$ & Combined optical efficiency & $1.5 \mathrm{Lm} / \mathrm{W}$ \\
\hline$\zeta$ & Attenuation coefficient & $10^{-6} \mathrm{~m}^{-1}$ \\
\hline$c$ & Speed of light & $3 \times 10^{8} \mathrm{~m} / \mathrm{s}$ \\
\hline$\alpha$ & Path loss exponent & 2 \\
\hline$l_{\mathrm{A}}$ & Atmospheric loss & $1.2 \mathrm{~dB}$ \\
\hline$\sigma^{2}$ & Variance of the AWGN & $-170 \mathrm{dBm}$ \\
\hline$\xi_{n}[k]$ & Data size of offloaded task & {$[1.7,2.1] \times 10^{6} \mathrm{bits}$} \\
\hline$B_{n}[k]$ & Total data size of task & {$[2.2,3.5] \times 10^{6} \mathrm{bits}$} \\
\hline$\kappa$ & Effective switched capacitance & $10^{-26}$ \\
\hline$\ell_{1}$ & Parameter related to flight model & $9.26 \times 10^{-3} \mathrm{~kg} / \mathrm{m}$ \\
\hline$\ell_{2}$ & Parameter related to flight model & $3500 \mathrm{~kg} \cdot \mathrm{m}^{3} / \mathrm{s}^{4}$ \\
\hline$m$ & Mass of all the payloads & $10 \mathrm{~kg}$ \\
\hline$g$ & Gravity acceleration & $9.8 \mathrm{~m} / \mathrm{s}^{2}$ \\
\hline
\end{tabular}

time horizon $T$. The HAP coverage space $\Omega$ is discretized into $Y=11$ tiers. All the tiers are evenly spaced by an equal vertical distance $L_{\mathrm{T}}=200 \mathrm{~m}$, and the vertical distance is set to $L_{0}=1 \mathrm{~km}$ between the tier 1 and the Earth's surface. In addition, each tier is composed of $500 \times 500$ square tile grids with equally sized areas, specified as $5 \mathrm{~m}^{2}$.

Regarding communication and computing models, unless otherwise mentioned, we set the equally-sized bandwidth of orthogonal subchannel for each AUE as $W=$ $25 \mathrm{MHz}$. For simplicity, the transmit power of each AUE to the HAP in A2A uplink transmission is assumed to be randomly assigned from the range of $[250 \mathrm{~mW}, 400 \mathrm{~mW}]$ at each time slot. We set the A2A uplink transmission overhead to be $\vartheta_{n}=50 \mathrm{bits}$ for each AUE. Furthermore, we assume that each AUE is flying at a constant speed during the chargingthen-MEC phase. Particularly, the velocity vector of each AUE is selected randomly from the range of $[(8,8),(15,20)]$ and the acceleration vector are always set to $\mathbf{a}_{n}[k]=(0,0)$. The positive weighting factors associated with the utility function in the proposed game framework are set to be $\lambda_{1}=0.97, \lambda_{2}=7 \times 10^{-4}$, and $\lambda_{3}=0.3$, respectively. The other relevant parameters adopted in simulations are summarized in Table 1

Before validating the system performance against the baseline schemes through the above simulation settings, we first provide insight on the effect of the number of battlefields and the tile grids distributed by two players for the proposed framework. Fig. 3(a) shows the effect of the number of battlefields $N$ on the expected total utilities of the defender with increasing values of $Z_{H}^{[T]}$ when $Z_{S}^{[T]}=200$. It is immediately seen that from this figure that the expected total utilities of the defender will gradually increase with the growth of the number of battlefields $N$ for a given $Z_{S}^{[T]}$. That is, the larger number of battlefields,

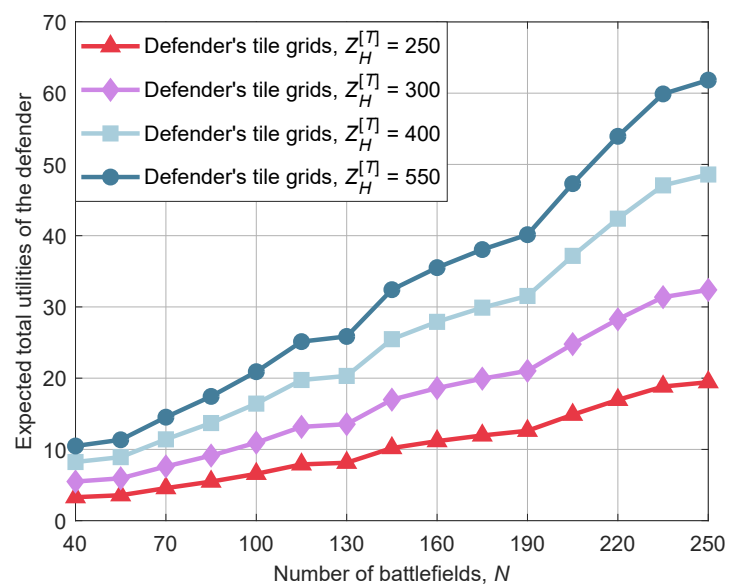

(a) $U_{H}^{[T]}\left(\mathbf{h}^{*}, \mathbf{s}^{*}\right)$ versus $N$, with $Z_{S}^{[T]}=200$.

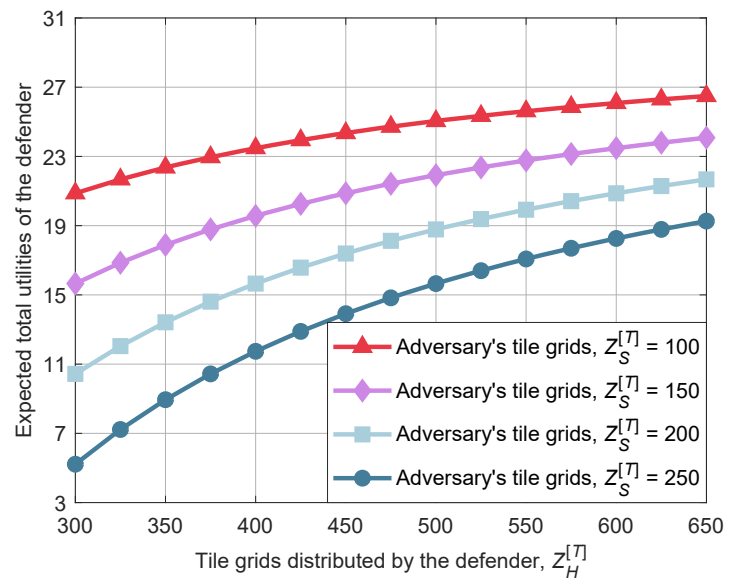

(b) $U_{H}^{[T]}\left(\mathbf{h}^{*}, \mathbf{s}^{*}\right)$ versus $Z_{H}^{[T]}$, with $N=100$.

Fig. 3. The effect of the number of battlefields $N$ and tile grids distributed by the defender $Z_{H}^{[T]}$ on the expected total utilities of the defender $U_{H}^{[T]}\left(\mathbf{h}^{*}, \mathbf{s}^{*}\right)$, when $f_{n}=4 \times 10^{9}$ cycles $/ \mathrm{s}, \varpi_{n}=110$ cycles $/ \mathrm{bit}$, $\beta=800 \mathrm{~W}$, and $\phi_{n}=1 \mathrm{GHz}$.

the more obtained expected total utilities of the defender for the system. Referring to Fig. 3(a), we can also observe that the defender with the higher value of $Z_{H}^{[T]}$ will obtain more expected total utilities by increasing the number of battlefields $N$. The main reason for this phenomenon is that the defender allocates the tile grids to more battlefields, and then can win in more battlefields to gain more expected total utilities. This result indicates that the choice of the number of battlefields and the battlefield resources of the defender has a negligible effect on the system performance and the expected total utilities of the proposed framework.

In Fig. 3(b), the expected total utilities of the defender is presented versus the tile grids distributed by the defender $Z_{H}^{[T]}$ by varying the tile grids allocated by the adversary 


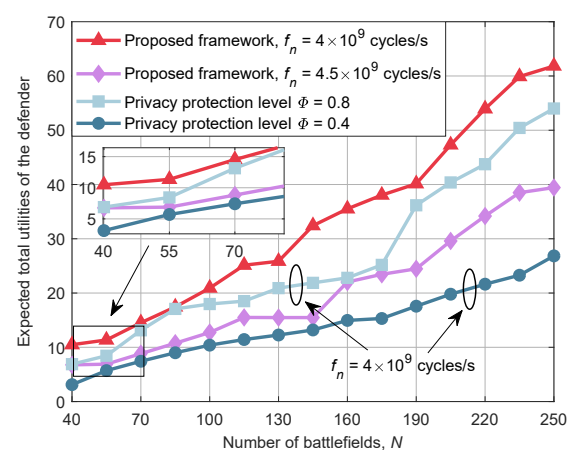

(a) $\beta=800 \mathrm{~W}$ and $\phi_{n}=1 \mathrm{GHz}$.

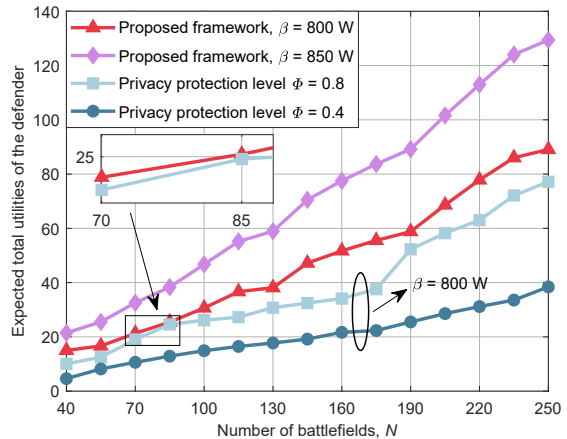

(b) $f_{n}=3.5 \times 10^{9}$ cycles $/ \mathrm{s}$ and $\phi_{n}=3 \mathrm{GHz}$

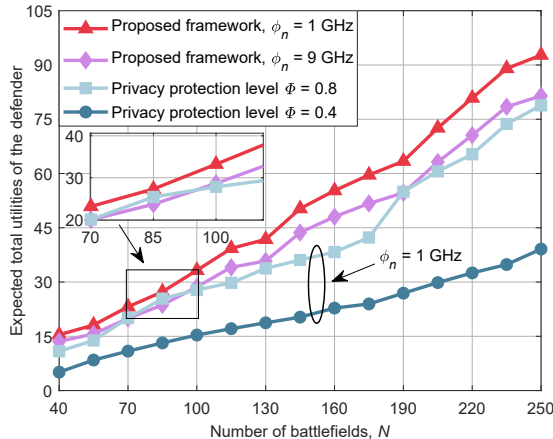

(c) $\beta=850 \mathrm{~W}$ and $f_{n}=4 \times 10^{9}$ cycles $/ \mathrm{s}$.

Fig. 4. Expected total utilities of the defender $U_{H}^{[T]}\left(\mathbf{h}^{*}, \mathbf{s}^{*}\right)$ versus number of battlefields $N$ for the proposed framework and two baselines with privacy protection level $\Phi=0.8$ and $\Phi=0.4$, respectively, when $Z_{H}^{[T]}=550, Z_{S}^{[T]}=200$, and $\varpi_{n}=110 \mathrm{cycles} / \mathrm{bit}$.

$Z_{S}^{[T]}$ when $N=100$. There was no difficulty to observe that with a larger value of $Z_{H}^{[T]}$, the global curve becomes gradually convergent and slowly rising. Especially, along with the increasing value of $Z_{H}^{[T]}$, the expected total utilities of the defender have been heightened for a given $Z_{S}^{[T]}$. Meanwhile, for a given $Z_{H}^{[T]}$, the more tile grids distributed by the adversary will generate the lower expected total utilities of the defender. This can be explained as follows: 1) With the increasing value of $Z_{H}^{[T]}$, the defender has more resources to counterbalance with the adversary, resulting in more expected total utilities obtained by the defender; and 2) for winning more battlefields, the adversary also reduces the expected total utilities of the defender by improving the value of $Z_{S}^{[T]}$ and competing with the defender. This result further provides a hint to choose the appropriate amount of battlefield resources for the defender to further improve the system performance.

Results in Fig. 4 show the comparison of the expected total utilities of the defender between the proposed framework and two baselines with privacy protection level $\Phi=0.8$ and $\Phi=0.4$, respectively, versus the number of battlefields $N$. Here, we assume that $Z_{H}^{[T]}=550$, $Z_{S}^{[T]}=200$, and $\varpi_{n}=110$ cycles/bit. In Fig. $4(\mathrm{a})$, we compare the expected total utilities of the defender between the proposed framework and two baselines under different local computing capabilities $f_{n}$ in the case of $\beta=800 \mathrm{~W}$ and $\phi_{n}=1 \mathrm{GHz}$. Overall, all the curves follow an upward trend with the increase of $N$. From Fig. 4(a), as for the proposed framework, it can be easily observed that the expected total utilities of the defender with a relatively lower value of $f_{n}$ are much higher than that with relatively higher value of $f_{n}$. This can be explained as follows: 1) A larger value of $f_{n}$ means the improvement of local computation capacity $R_{n}$ as shown in (18); and 2) the more energy consumption for local computing of AUE is required to locally accomplish the computation task, leading the lower expected total utilities of the defender. In contrast, for a given $f_{n}$, the proposed framework obviously outperforms two baselines no matter how many battlefields are applied. This is not much surprising since a smaller proportion of the number of the battlefields with the enhanced location privacy, i.e., the privacy protection level $\Phi$, using the proposed framework incurs much lower expected total utilities of the defender.

Fig. 4(b) depicts the effect of number of battlefields $N$ on the expected total utilities of the defender for the proposed framework and two baselines with privacy protection level $\Phi=0.8$ and $\Phi=0.4$, respectively, when $f_{n}=3.5 \times 10^{9} \mathrm{cycles} / \mathrm{s}$ and $\phi_{n}=3 \mathrm{GHz}$. Here, we set the optical laser power of the laser beam at the HAP as $\beta=800 \mathrm{~W}$ for two baselines. For comparison, we employ $\beta=850 \mathrm{~W}$ and $\beta=800 \mathrm{~W}$ for the proposed framework, respectively. As expected, all the curves in this figure grow up with the increase of $N$. By increasing the value of $\beta$, we can further find that the expected total utilities of the defender will be significantly improved for the proposed framework. This behavior is explained by the fact that the AUEs can obtain more harvested energy $E_{n}^{\mathrm{H}}$ to accomplish the computation task, which not only enhances the local computation capacity $R_{n}$, but also leads to the ever-growing expected total utilities for the system. Referring to this result, it can be also seen that the proposed framework outperforms two baselines with respect to the expected total utilities of the defender. This is mainly because the privacy protection level $\Phi$ of the proposed framework is much bigger than that of the two baselines, incurring more battlefields won by the defender and thus more expected total utilities obtained by the defender.

In Fig. 4(c), we plot the expected total utilities of the defender versus number of battlefields $N$ for the proposed framework and two baselines with privacy protection level $\Phi=0.8$ and $\Phi=0.4$, respectively, with the adjusted carrier frequency $\phi_{n}$ in the case of $\beta=850 \mathrm{~W}$ and $f_{n}=$ $4 \times 10^{9} \mathrm{cycles} / \mathrm{s}$. We observe that it becomes more difficult to obtain the high expected total utilities by increasing the value of $\phi_{n}$ in the proposed framework. On the contrary, it is easier to get higher expected total utilities at a lower value of $\phi_{n}$ in the proposed framework. This is due to the fact that the carrier frequency of subchannel for the AUE is related to its achievable uplink rate $R_{n}^{\mathrm{U}}[k]$ at each time slot. Obviously, the larger value of $\phi_{n}$ results in the smaller channel gain $g_{n}[k]$, which therefore generates the lower achievable uplink rate $R_{n}^{\mathrm{U}}[k]$. Such a case can further cause the reduced computation task offloaded to the HAP, thus resulting in the lower expected total utilities of the defender. Referring to this result, we also find that the proposed framework 


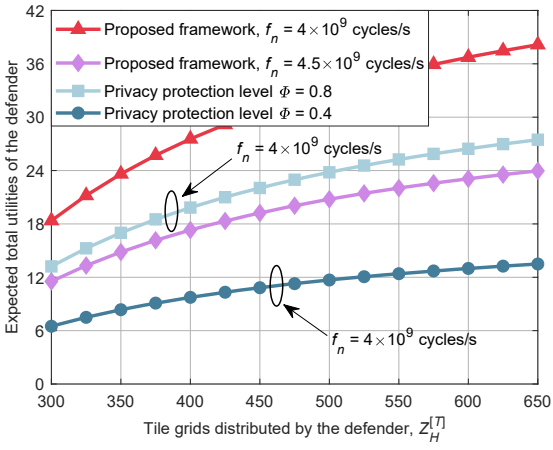

(a) $\beta=800 \mathrm{~W}$ and $\phi_{n}=1 \mathrm{GHz}$.

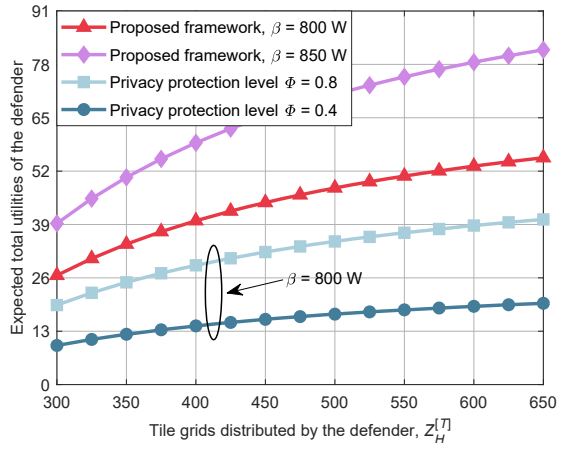

(b) $f_{n}=3.5 \times 10^{9}$ cycles $/ \mathrm{s}$ and $\phi_{n}=3 \mathrm{GHz}$.

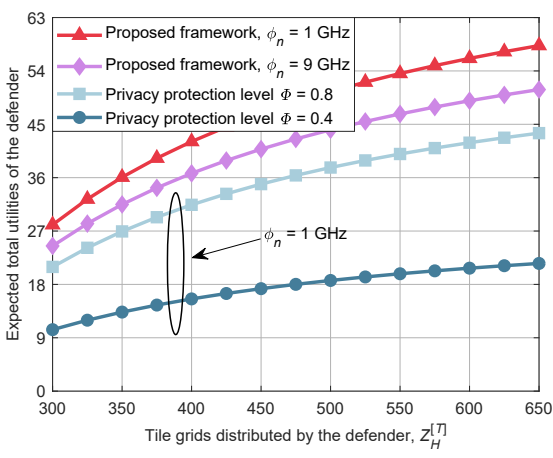

(c) $\beta=850 \mathrm{~W}$ and $f_{n}=4 \times 10^{9}$ cycles $/ \mathrm{s}$.

Fig. 5. Expected total utilities of the defender $U_{H}^{[T]}\left(\mathbf{h}^{*}, \mathbf{s}^{*}\right)$ versus tile grids distributed by the defender $Z_{H}^{[T]}$ for the proposed framework and two baselines with privacy protection level $\Phi=0.8$ and $\Phi=0.4$, respectively, when $Z_{S}^{[T]}=200, N=150$, and $\varpi_{n}=110 \mathrm{cycles} / \mathrm{bit}$.

outperforms two baselines with privacy protection level $\Phi=0.8$ and $\Phi=0.4$ in terms of the expected total utilities of the defender under a given value of $\phi_{n}$.

Fig. 5 presents the comparison of the expected total utilities of the defender between the proposed framework and two baselines with privacy protection level $\Phi=0.8$ and $\Phi=0.4$, respectively, versus the tile grids distributed by the defender $Z_{H}^{[T]}$, when $Z_{S}^{[T]}=200, N=150$, and $\varpi_{n}=$ 110 cycles/bit. In Fig. 5(a), we illustrate the expected total utilities of the defender between the proposed framework and two baselines with varying local computing capabilities $f_{n}$. Here, for comparison purpose, we set $\beta=800 \mathrm{~W}$ and $\phi_{n}=1 \mathrm{GHz}$. It is evident from this figure that the expected total utilities of the defender markedly increases with the continuous evolution of tile grids distributed by the defender $Z_{H}^{[T]}$. This is because by increasing the value of $Z_{H}^{[T]}$, the defender owns more battlefield resources to compete with the adversary and therefore obtains higher expected total utilities of the system. We can also observe that a higher value of $Z_{H}^{[T]}$ can further ensure the continued growth of the expected total utilities of the defender although the privacy protection level $\Phi$ decreases gradually. Furthermore, as observed from Fig. 5(a), it would be preferable to decrease the value of $f_{n}$ to gain more expected utilities of the defender. This is due to that although the increase of the value of $f_{n}$ reduces the computation delay for local computing $T_{n}^{\mathrm{L}}$, the energy consumption for local computing $E_{n}^{\mathrm{L}}$ has been accordingly improved. Such a result may further increase the system burden and reduce the expected total utilities of the defender.

By adjusting the parameter of optical laser power $\beta$ from $800 \mathrm{~W}$ to $850 \mathrm{~W}$, Fig. 5(b) compares the expected total utilities of the defender between the proposed framework and two baselines with privacy protection level $\Phi=0.8$ and $\Phi=0.4$, respectively, when $f_{n}=3.5 \times 10^{9}$ cycles $/ \mathrm{s}$ and $\phi_{n}=3 \mathrm{GHz}$. As expected, if the privacy protection level $\Phi$ continuously increases, the expected total utilities of the defender will increase accordingly no matter what the value of $Z_{H}^{[T]}$ is. This can be explained as follows: 1) The AUEs can harvest more energy to complete the computation tasks due to the improved privacy protection level $\Phi$, and 2) the local computing capacity $R_{n}$ can be also increased to improve the expected total utilities of the defender when the value of $\beta$ is incorporated into the proposed framework.

Fig. 5(c) illustrates the expected total utilities of the defender versus the tile grids distributed by the defender for the proposed framework and two baselines with privacy protection level $\Phi=0.8$ and $\Phi=0.4$, respectively, by adjusting carrier frequency $\phi_{n}$ when $\beta=850 \mathrm{~W}$ and $f_{n}=4 \times 10^{9}$ cycles $/ \mathrm{s}$. As can be seen in Fig. 5(c) the proposed framework with a smaller value of $\phi_{n}$ can obtain better utilities specifically when we increase the number of defender's tile grids. On the contrary, by applying larger value of $\phi_{n}$, both the channel gain $g_{n}[k]$ and the achievable uplink rate $R_{n}^{\mathrm{U}}[k]$ will decrease. Such result may generate more uplink transmission delay $T_{n}^{\mathrm{U}}$ and energy consumption $E_{n}^{\mathrm{U}}$ for each AUE to offload the computation tasks to the HAP. Thereby, the expected total utilities of the defender are decreased. For a given value of $\phi_{n}$, the expected total utilities of the defender of the proposed framework are higher than that of two baselines with privacy protection level $\Phi=0.8$ and $\Phi=0.4$, respectively. To explain, the smaller the privacy protection level is, the smaller proportion of the number of AUEs with the enhanced location privacy for laser WPT, leading to the reduced number of battlefields the defender wins.

Finally, in Fig. 6, we illustrate the expected total utilities of the defender versus tile grids distributed by two players for the proposed framework and two baselines with privacy protection level $\Phi=0.8$ and $\Phi=0.4$, respectively, in the case of $\beta=800 \mathrm{~W}, \phi_{n}=1 \mathrm{GHz}$, and $f_{n}=4 \times 10^{9}$ cycles $/ \mathrm{s}$. From Fig. 6(a), Fig. 6(b) and Fig. 6(c) as a whole, we easily observe that the increase of the number of battlefields $N$ drastically affects the expected total utilities of the defender achieved by the proposed framework and two baselines, respectively. It can be also seen that the baseline with privacy protection level $\Phi=0.8$ can achieve a near-optimal performance close to that with the proposed framework. Moreover, for a given number of battlefields $N$, the lower value of $\varpi_{n}$ results in a significant utility improvement compared to the higher value of $\varpi_{n}$ in the proposed framework. The intuition behind the observation is the fact that the AUEs require more CPU cycles to process one bit of raw data, resulting in fewer computation tasks locally executed during a fixed time horizon, and thereupon reducing the 


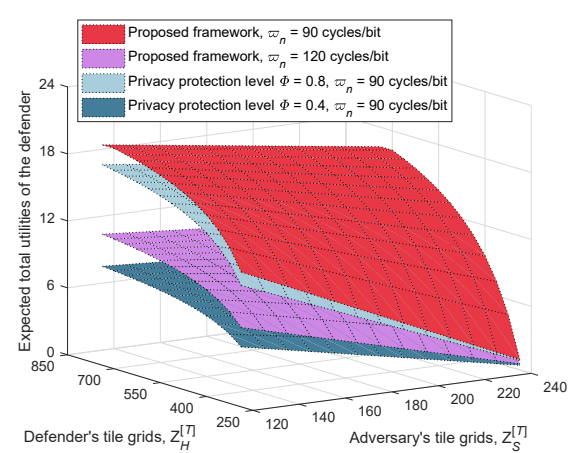

(a) $N=50$.

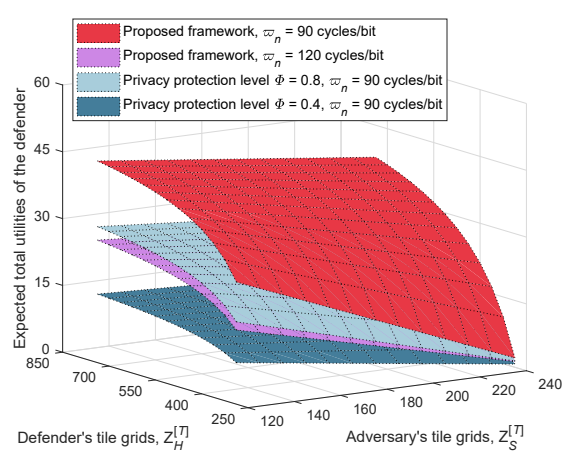

(b) $N=100$.

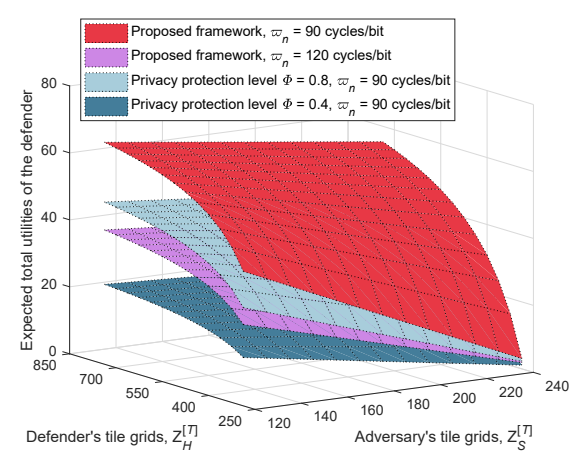

(c) $N=150$

Fig. 6. Expected total utilities of the defender $U_{H}^{[T]}\left(\mathbf{h}^{*}, \mathbf{s}^{*}\right)$ versus tile grids distributed by two players, i.e., $Z_{H}^{[T]}$ and $Z_{S}^{[T]}$, for the proposed framework and two baselines with privacy protection level $\Phi=0.8$ and $\Phi=0.4$, respectively, when $\beta=800 \mathrm{~W}, \phi_{n}=1 \mathrm{GHz}$, and $f_{n}=4 \times 10^{9} \mathrm{cycles} / \mathrm{s}$.

local computation capacity $R_{n}$ for each AUE. Therefore, the expected total utilities of the defender are clearly inversely related to $\varpi_{n}$. Such observations above bolster the importance of choosing an appropriate number of CPU cycles to process one bit of raw data at the AUE for improving the expected total utilities of the system.

\section{Conclusion}

In this paper, we proposed the privacy-aware laser-powered aerial MEC framework, specifically designed for addressing the new privacy vulnerability arising from the WPT signaling transmission in the presence of the terrestrial adversary. This framework was aimed at the realization of a tradeoff between privacy preservation in laser WPT and system performance in total utilities to secure the HAP-aided MEC systems under the CLDF attacks mounted by the adversary. To determine the aerial charging locations for AUEs, we designed the multi-tier tile grid-based spatial structure through the discretization of the $3 \mathrm{D}$ coverage space of the HAP. On this basis, to enhance the charging location privacy of AUEs, we formulated the interaction between the HAP as the defender and the adversary in their allocation of the limited number of tile grids as charging locations to the AUEs as the Colonel Blotto game. This game framework characterized the competition of two players for limited resources over a finite set of battlefields during a specific time horizon. The utility function that each player receives from an individual battlefield in this tile grid allocation game was specified by capturing the balance among the local computation capacity, the energy consumption, and the execution delay of each AUE. Then, the mixed-strategy Nash equilibria of this game were derived under the constraints of symmetric and asymmetric tile grids between the defender and the adversary, respectively.

Simulation results demonstrated the effectiveness and practicality of our proposed framework, which achieves good performance with the beneficial increase on the system-wide expected total utilities compared with other baseline schemes. The theoretical analyses and simulation results offered valuable design insights for practical laserpowered aerial MEC systems with enhanced security and privacy.

\section{APPENDIX A PROOF OF THEOREM 1}

Due to the symmetric players as mentioned, the tile grid allocation game $\mathfrak{C B}_{2}$ with mixed strategy can be formulated by a Colonel Blotto game with symmetric resources across battlefield set $\mathcal{N}$. In this case, the battlefield resources allocated by the defender are the same as that of the adversary. That is, the total number of tile grids that can be distributed by two players are completely equal, i.e., $Z_{H}^{[T]}=Z_{S}^{[T]}$.

For the defender, the ultimate goal is to ensure that more AUEs obtain the successful laser WPT and then perform the aerial MEC for their computation tasks. By optimally allocating its defense resources, the defender obtains the associated expected utilities. In contrast, the adversary aims to prevent more AUEs from receiving successful laser WPT by allocating its attack resources and obtaining the expected utilities. Therefore, it is uncovered that regardless of the purpose of two players, the price paid on each battlefield belongs to the same value. That is, the value of each battlefield is equal for two players, i.e., the total data size of computation task during time horizon $T$. For analytical tractability, the value of battlefield $n$ for both players is thus defined as the total data size of computation task for AUE $n$ during time horizon $T$, i.e., $B_{n}$. And, the total value of $N$ battlefields for both players is then given by $\widehat{B}=\sum_{n=1}^{N} B_{n}$.

By the aid of the battlefield value setup, the mixedstrategy Colonel Blotto game has an NE $\left(\mathbf{h}^{*}, \mathbf{s}^{*}\right)$, where $\mathbf{h}^{*}$ (or $\mathbf{s}^{*}$ ) is the uniform distribution probability vector of the tile grid distribution vector $\mathbf{H}$ (or $\mathbf{S}$ ). Moreover, the number of tile grids distributed by the defender (or the adversary) to battlefield $n$ is denoted by $H_{n}^{*}$ (or $S_{n}^{*}$ ). Since the total number of tile grids allocated by the defender and the adversary are completely equal, both players will distribute the tile grids reasonably to each battlefield for wining more battlefields. Therefore, the number of tile grids allocated by both players for battlefield $n$ are also equal at the mixedstrategy NE, i.e., $H_{n}^{*}=S_{n}^{*}$.

According to Proposition 1 in [39], $H_{n}^{*}$ and $S_{n}^{*}$ are uniformly distributed on $\left[0,\left|2 Z_{H}^{[T]} B_{n} / \widehat{B}\right|\right]$. By combining (16) and (17), we can obtain the uniform distribution vector $\mathbf{H}^{*}$ (or $\mathbf{S}^{*}$ ) of the defender (or the adversary) to battlefield $n$ under the derived mixed-strategy NE with symmetric 
players as follows

$$
\begin{aligned}
\mathbf{H}^{*}=\mathbf{S}^{*} & =\left\{H_{n}^{*}, S_{n}^{*}\right\} \\
& =\mathscr{U}\left(\left\{0,1,2, \cdots,\left\lfloor\frac{2 Z_{H}^{[T]} B_{n}}{\widehat{B}}\right\rfloor\right\}\right),
\end{aligned}
$$

where $\mathscr{U}(\cdot)$ denotes the uniform distribution of the elements.

Due to the symmetric resources and the similar uniform distribution interval of both players, by combining (25), (26, and $(36)$, the uniform distribution probability $\mathbf{h}^{*}$ (or $\mathbf{s}^{*}$ ) of the defender (or the adversary) to battlefield $n$ under the derived mixed-strategy NE with symmetric players is also completely equal when $r \in\left[0, Z_{H}^{[T]}\right]$, which can be determined as

$$
\begin{aligned}
& \mathbf{h}^{*}= \mathbf{s}^{*}=\left\{H_{n, r}^{*}, S_{n, r}^{*}\right\} \\
&= \begin{cases}\frac{1}{\left\lfloor\frac{2 Z_{H}^{[T]} B_{n}}{\widehat{B}}\right\rfloor+1}, & r \in\left[0,\left\lfloor\frac{2 Z_{H}^{[T]} B_{n}}{\widehat{B}}\right\rfloor\right], \\
0, & r \in\left(\left\lfloor\frac{2 Z_{H}^{[T]} B_{n}}{\widehat{B}}\right\rfloor, Z_{H}^{[T]}\right] .\end{cases}
\end{aligned}
$$

For notational simplicity, let $\Upsilon=Z_{H}^{[T]} / \widehat{B}$. According to (37), the uniform distribution probability for battlefield $n$ of both players are given by $H_{n, r}^{*}=S_{n, r}^{*}$, for any value of the number of allocated tile grids $r$. When $r \in\left[0,\left\lfloor 2 \Upsilon B_{n}\right\rfloor\right]$, the defender (or the adversary) has $\left\lfloor 2 \Upsilon B_{n}\right\rfloor+1$ schemes of tile grids allocation for battlefield $n$, and the unifor$\mathrm{m}$ distribution probability of each scheme is equal, i.e., $1 /\left(\left\lfloor 2 \Upsilon B_{n}\right\rfloor+1\right)$. To characterize the set of the real numbers of the uniform distribution probability for both players to battlefield $n$, we employ a matrix of $1 \times\left(\left\lfloor 2 \Upsilon B_{n}\right\rfloor+1\right)$, where each element is given as $\frac{1}{\left[2 \Upsilon B_{n}\right\rfloor+1}$. The result is also a allone matrix, i.e., $\frac{1}{\left\lfloor 2 \Upsilon B_{n}\right\rfloor+1} \mathbf{1}_{1 \times\left(\left\lfloor 2 \Upsilon B_{n}\right\rfloor+1\right)}$.

Similarly, when $r \in\left(\left\lfloor 2 \Upsilon B_{n}\right\rfloor, Z_{H}^{[T]}\right]$, the defender (or the adversary) has $Z_{H}^{[T]}-\left\lfloor 2 \Upsilon B_{n}\right\rfloor$ schemes of tile grid allocation to battlefield $n$. However, the uniform distribution vector $\mathbf{h}^{*}$ (or $\mathbf{s}^{*}$ ) does not exist, which is always zero. Next, we can further formulate a matrix of $1 \times\left(Z_{H}^{[T]}-\left\lfloor 2 \Upsilon B_{n}\right\rfloor\right)$ to denote the set of the real numbers relevant to the $\mathrm{u}$ niform distribution probability assigned by both players to battlefield $n$. Since each element in such a matrix is zero, the matrix also belongs to an all-zero matrix, i.e., $\mathbf{0}_{1 \times\left(Z_{H}^{[T]}-\left\lfloor 2 \Upsilon B_{n}\right\rfloor\right)}$. Combining the all-one and all-zero matrices as derived together, we finally obtain the matrix of 29, which completes the proof.

\section{APPENDIX B}

\section{PROOF OF THEOREM 2}

Depending on the asymmetric players, the tile grid allocation game $\mathfrak{C B}_{2}$ with mixed strategy can be formulated as a Colonel Blotto game with asymmetric resources over battlefield set $\mathcal{N}$. The mixed-strategy Colonel Blotto game game has a $\mathrm{NE}\left(\mathbf{h}^{*}, \mathbf{s}^{*}\right)$, where $\mathbf{h}^{*}\left(\right.$ or $\left.\mathbf{s}^{*}\right)$ is the distribution probability in the distribution vector $\mathbf{H}$ (or $\mathbf{S}$ ). As stated in [34], the defender (or the adversary) chooses the probability density function $\mathcal{Z}_{n}^{H}\left(H_{n}^{*}\right)$ (or $\mathcal{Z}_{n}^{S}\left(S_{n}^{*}\right)$ ) according to the allocated resource budget of tile grids, for $H_{n}^{*}, S_{n}^{*} \in\left[0,\left\lfloor 2 Z_{H}^{[T]} / N\right\rfloor\right]$, which can be calculated as

$$
\mathcal{Z}_{n}^{H}\left(H_{n}^{*}\right)=\frac{H_{n}^{*}}{\left\lfloor\frac{2 Z_{H}^{[T]}}{N}\right\rfloor},
$$

$$
\mathcal{Z}_{n}^{S}\left(S_{n}^{*}\right)=\left(1-\frac{Z_{S}^{[T]}}{Z_{H}^{[T]}}\right) \mathscr{F}\left(S_{n}^{*}\right)+\left(\frac{Z_{S}^{[T]}}{Z_{H}^{[T]}}\right) \frac{S_{n}^{*}}{\left[\frac{2 Z_{H}^{[T]}}{N}\right]},
$$

where $\mathscr{F}(\cdot)$ corresponds to the unit pulse function. That is, if $S_{n}^{*}=0, \mathscr{F}\left(S_{n}^{*}\right)=1$; if $S_{n}^{*} \in\left[1,\left|2 Z_{H}^{[T]} / N\right|\right], \mathscr{F}\left(S_{n}^{*}\right)=0$.

By combining (16) and (38), we comprehended that the number of tile grids assigned by the defender to battlefield $n$ is distributed in the interval $H_{n}^{*} \in\left[0,\left|2 Z_{H}^{[T]} / N\right|\right]$. Hence, the uniform distribution vector $\mathbf{H}^{*}$ of the defender to battlefield $n$ under the mixed-strategy NE with asymmetric players is thus expressed by

$$
\mathbf{H}^{*}=\left\{H_{n}^{*}\right\} \sim \mathscr{U}\left(\left\{0,1,2, \cdots,\left\lfloor\frac{2 Z_{H}^{[T]}}{N}\right\rfloor\right\}\right) .
$$

Note that $\mathscr{U}(\cdot)$ corresponds to the uniform distribution of the elements. Similarly, invoking (17) and (39), the number of tile grids assigned by the adversary to battlefield $n$ is also distributed in the interval $S_{n}^{*} \in\left[0,\left|2 Z_{H}^{[T]} / N\right|\right]$. For the interval as considered, there are two cases, i.e., $S_{n}^{*}=0$ and $S_{n}^{*} \neq 0$. Thus, we can now arrive at the uniform distribution vector $\mathbf{S}^{*}$ of the adversary to battlefield $n$ as follows

$$
\begin{aligned}
\mathbf{S}^{*}=\left\{S_{n}^{*}\right\} & \sim\left(1-\frac{Z_{S}^{[T]}}{Z_{H}^{[T]}}\right) \mathscr{F}\left(S_{n}^{*}\right)+\left(\frac{Z_{S}^{[T]}}{Z_{H}^{[T]}}\right) \\
& \left.\times \mathscr{U}\left(\left\{0,1,2, \cdots, \mid \frac{2 Z_{H}^{[T]}}{N}\right\rfloor\right\}\right) .
\end{aligned}
$$

Recall that the relationship associated with the total number of tile grids distributed between the defender and the adversary should be $Z_{H}^{[T]}>Z_{S}^{[T]}$ over battlefield set $\mathcal{N}$ for the tile grid allocation game $\mathfrak{C B}_{2}$. Thus, the number of tile grids uniformly allocated to battlefield $n$ by the defender can be interpreted as $r \in\left[1,\left|2 Z_{H}^{[T]} / N\right|\right]$. As in $|35|$, based on 25 and $(40)$, the uniform distribution probability $\mathbf{h}^{*}$ of the defender to battlefield $n$ under the mixed-strategy NE with asymmetric players can be determined by

$$
\begin{aligned}
\mathbf{h}^{*} & =\left\{H_{n, r}^{*}\right\} \\
& = \begin{cases}0 & r=0 \\
\frac{1}{\left\lfloor\frac{2 Z_{H}^{[T]}}{N}\right\rfloor}, & r \in\left[1,\left\lfloor\frac{2 Z_{H}^{[T]}}{N}\right\rfloor\right], \\
0, & r \in\left(\left\lfloor\frac{2 Z_{H}^{[T]}}{N}\right\rfloor, Z_{H}^{[T]}\right] .\end{cases}
\end{aligned}
$$


Meanwhile, by using (26) and (41), we can further derive the uniform distribution probability $\mathbf{s}^{*}$ of the adversary to battlefield $n$, which is specified as

$$
\begin{aligned}
& \mathbf{s}^{*}=\left\{S_{n, r}^{*}\right\}
\end{aligned}
$$

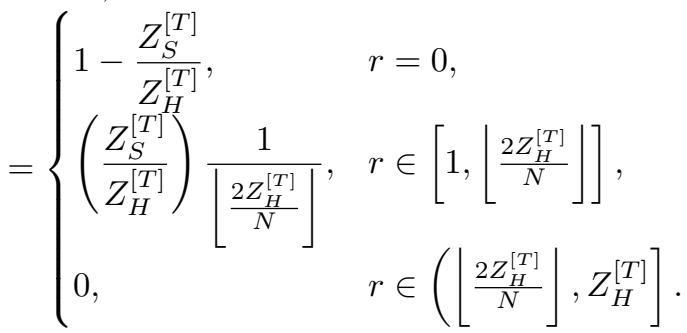

For notational simplicity, let $\Xi=Z_{H}^{[T]} / N$. According to (42), the defender has one scheme of tile grid allocation for each battlefield when $r=0$, and the uniform distribution probability $\mathbf{h}^{*}$ of each battlefield is always zero. Thereby, we represent the set of the real numbers of the uniform distribution probability for $N$ battlefields as a matrix of $N \times 1$, wherein each element is zero, i.e., an all-zero matrix, denoted by $\mathbf{0}_{N \times 1}$.

When $r \in[1,\lfloor 2 \Xi\rfloor]$, it has $\lfloor 2 \Xi\rfloor$ schemes of tile grids uniformly allocated by the defender to each battlefield, and the uniform distribution probability of each scheme is equal, i.e., $\mathbf{h}^{*}=\frac{1}{[2 \Xi]}$. Then we characterize the set of the real numbers of the uniform distribution probability for $N$ battlefields by a matrix of $N \times\lfloor 2 \Xi\rfloor$, wherein each element is $\frac{1}{\lfloor 2 \Xi\rfloor}$, i.e., an all-one matrix, denoted as $\frac{1}{\lfloor 2 \Xi\rfloor} \mathbf{1}_{N \times\lfloor 2 \Xi\rfloor}$.

When $r \in\left(\lfloor 2 \Xi\rfloor, Z_{H}^{[T]}\right]$, the defender can uniformly allocate the tile grids for each battlefield through $Z_{H}^{[T]}-\lfloor 2 \Xi\rfloor$ schemes, and accordingly the uniform distribution probability $\mathbf{h}^{*}$ is always zero. Then we model the set of the real numbers of the uniform distribution probability for $N$ battlefields as a matrix of $N \times\left(Z_{H}^{[T]}-\lfloor 2 \Xi\rfloor\right)$, which also refers to an all-zero matrix, denoted by $\mathbf{0}_{N \times\left(Z_{H}^{[T]}-\lfloor 2 \Xi\rfloor\right)}$. Combining the all-one and all-zero matrices as derived, we obtain the matrix of 32.

Similarly, for 43 , the adversary allocates zero tile grid to each battlefield when $r=0$ with the uniform distribution probability $\mathbf{s}^{*}=1-Z_{S}^{[T]} / Z_{H}^{[T]}$. We denote the set of the real numbers of the uniform distribution probability for $N$ battlefields as a matrix of $N \times 1$, wherein each element is $1-Z_{S}^{[T]} / Z_{H}^{[T]}$, i.e., an all-one matrix, defined by $\left(1-Z_{S}^{[T]} / Z_{H}^{[T]}\right) \mathbf{1}_{N \times 1}$.

When $r \in[1,\lfloor 2 \Xi\rfloor]$, the adversary has $\lfloor 2 \Xi\rfloor$ schemes to allocate the tile grids uniformly over each battlefield, and the uniform distribution probability of each scheme is equal, i.e., $\mathbf{s}^{*}=Z_{S}^{[T]} / Z_{H}^{[T]}\lfloor 2 \Xi\rfloor$. Then we use a matrix of $N \times\lfloor 2 \Xi\rfloor$ to express the set of the real numbers of the uniform distribution probability for $N$ battlefields. Specifically, such a matrix is an all-one matrix written by $\left(Z_{S}^{[T]} / Z_{H}^{[T]}\lfloor 2 \Xi\rfloor\right) \mathbf{1}_{N \times\lfloor 2 \Xi\rfloor}$.

When $r \in\left(\lfloor 2 \Xi\rfloor, Z_{H}^{[T]}\right]$, the adversary has $Z_{H}^{[T]}-\lfloor 2 \Xi\rfloor$ schemes to uniformly assign the tile grids to $N$ battlefields, and the uniform distribution probability $\mathbf{s}^{*}$ of each scheme always is zero. We thus denote the set of the real numbers of the uniform distribution probability for $N$ battlefields as a matrix of $N \times\left(Z_{H}^{[T]}-\lfloor 2 \Xi\rfloor\right)$, which is an all-zero matrix, represented by $\mathbf{0}_{N \times\left(Z_{H}^{[T]}-\lfloor 2 \Xi\rfloor\right)}$. Based on the derived all-one and all-zero matrices, the matrix of 33 is finally obtained. This completes the proof.

\section{ACKNOWLEDGMENTS}

This work was supported in part by the National Natural Science Foundation of China under Grants 61971032 and 61901115, in part by the Natural Science Foundation of Hebei Province under Grants F2019402206, A2020402013, and F2018402198, in part by the Research Program for Top-notch Young Talents in Hebei Province under Grant BJ2017037, in part by the Fundamental Research Funds for Central Universities under Grants FRF-TP-18-008A3 and 328201911, and in part by the U.S. National Natural Science Foundation under Grants EARS-1839818, CNS1717454, CNS-1731424, and CNS-1702850.

\section{REFERENCES}

[1] Y. Wang, L. Zhang, M. Min, C. Guo, V. Sharma, and Z. Han, "Privacyaware wireless power transfer for aerial computation offloading via colonel blotto game," in Proc. IEEE Globecom Workshops, Taipei, Taiwan, Dec. 2020.

[2] Y. Zeng, J. Lyu, and R. Zhang, "Cellular-connected UAV: Potential, challenges, and promising technologies," IEEE Wireless Commun., vol. 26, no. 1, pp. 120-127, Feb. 2019.

[3] M. Mozaffari, A. T. Z. Kasgari, W. Saad, M. Bennis, and M. Debbah, "Beyond 5G with UAVs: Foundations of a 3D wireless cellular network," IEEE Trans. Wireless Commun., vol. 18, no. 1, pp. 357372, Jan. 2019.

[4] L. Zhang, H. Zhao, S. Hou, Z. Zhao, H. Xu, X. Wu, Q. Wu, and R. Zhang, "A survey on $5 \mathrm{G}$ millimeter wave communications for UAV-assisted wireless networks," IEEE Access, vol. 7, pp. 117 460117 504, Jul. 2019.

[5] U. Challita, A. Ferdowsi, M. Chen, and W. Saad, "Machine learning for wireless connectivity and security of cellular-connected UAVs," IEEE Wireless Commun., vol. 26, no. 1, pp. 28-35, Feb. 2019.

[6] M. Erdelj, E. Natalizio, K. R. Chowdhury, and I. F. Akyildiz, "Help from the sky: Leveraging UAVs for disaster management," IEEE Pervasive Comput., vol. 16, no. 1, pp. 24-32, Jan. 2017.

[7] J. Moura and D. Hutchison, "Game theory for multi-access edge computing: Survey, use cases, and future trends," IEEE Commun. Surv. Tutor., vol. 21, no. 1, pp. 260-288, First Quarter 2019.

[8] L. Zhang, H. Zhang, C. Guo, H. Xu, L. Song, and Z. Han, "Satelliteaerial integrated computing in disasters: User association and offloading decision," in Proc. IEEE ICC, Dublin, Ireland, Jun. 2020.

[9] W. Jaafar and H. Yanikomeroglu, "Dynamics of laser-charged UAVs: A battery perspective," IEEE Internet Things J., 2020, Early Access.

[10] K. Jin and W. Zhou, "Wireless laser power transmission: A review of recent progress," IEEE Trans. Power Electron., vol. 34, no. 4, pp. 3842-3859, Apr. 2019.

[11] X. Lu, P. Wang, D. Niyato, D. I. Kim, and Z. Han, "Wireless charging technologies: Fundamentals, standards, and network applications," IEEE Commun. Surv. Tutor., vol. 18, no. 2, pp. 14131452, Second Quarter 2016.

[12] J. Ouyang, Y. Che, J. Xu, and K. Wu, "Throughput maximization for laser-powered UAV wireless communication systems," in Proc. IEEE ICC Workshops, Kansas City, MO, May 2018.

[13] M.-M. Zhao, Q. Shi, and M.-J. Zhao, "Efficiency maximization for UAV-enabled mobile relaying systems with laser charging," IEEE Trans. Wireless Commun., vol. 19, no. 5, pp. 3257-3272, May 2020.

[14] M. Mori, H. Kagawa, and Y. Saito, "Summary of studies on space solar power systems of Japan Aerospace Exploration Agency (JAXA)," Acta Astronautica, vol. 59, no. 1-5, pp. 132-138, Jul. 2006.

[15] Q. Liu, K. S. Yildirim, P. Pawelczak, and M. Warnier, "Safe and secure wireless power transfer networks: Challenges and opportunities in RF-based systems," IEEE Commun. Mag., vol. 54, no. 9, pp. 74-79, Sep. 2016. 
[16] J. Feng, Q. Pei, F. R. Yu, X. Chu, and B. Shang, "Computation offloading and resource allocation for wireless powered mobile edge computing with latency constraint," IEEE Wirel. Commun. Lett., vol. 8, no. 5, pp. 1320-1323, Oct. 2019.

[17] S. Mao, S. Leng, S. Maharjan, and Y. Zhang, "Energy efficiency and delay tradeoff for wireless powered mobile-edge computing systems with multi-access schemes," IEEE Trans. Wireless Commun., vol. 19, no. 3, pp. 1855-1867, Mar. 2020.

[18] Y. Liu, K. Xiong, Q. Ni, P. Fan, and K. B. Letaief, "UAV-assisted wireless powered cooperative mobile edge computing: Joint offloading, CPU control, and trajectory optimization," IEEE Internet Things J., vol. 7, no. 4, pp. 2777-2790, Apr. 2020.

[19] M.-A. Lahmeri, M. A. Kishk, and M.-S. Alouini, "Stochastic geometry-based analysis of airborne base stations with laserpowered UAVs," IEEE Commun. Lett., vol. 24, no. 1, pp. 173-177, Jan. 2020.

[20] M. Z. Hassan, M. J. Hossain, J. Cheng, and V. C. M. Leung, "Statistical-QoS guarantee for IoT network driven by laserpowered UAV relay and RF backscatter communications," IEEE Trans. Green Commun. Netw., vol. 5, no. 1, pp. 406-425, Mar. 2021.

[21] Q. Zhang, W. Fang, Q. Liu, J. Wu, P. Xia, and L. Yang, “Distributed laser charging: A wireless power transfer approach," IEEE Internet Things J., vol. 5, no. 5, pp. 3853-3864, Oct. 2018.

[22] L. Jiang, S. Xie, S. Maharjan, and Y. Zhang, "Blockchain empowered wireless power transfer for green and secure internet of things," IEEE Network, vol. 33, no. 6, pp. 164-171, Nov. 2019.

[23] L. Jiang, B. Chen, S. Xie, S. Maharjan, and Y. Zhang, "Incentivizing resource cooperation for blockchain empowered wireless power transfer in UAV networks," IEEE Trans. Veh. Technol., vol. 69, no. 12, pp. 15828-15841, Dec. 2020.

[24] Z. Zhang, K. T. Chau, C. Liu, C. Qiu, and F. Lin, “An efficient wireless power transfer system with security considerations for electric vehicle applications," Journal of Applied Physics, vol. 115, no. 17, p. 17A328, May 2014.

[25] L. Qian, E. W. Chan, P. P. Lee, and C. He, "Characterization of 3G control-plane signaling overhead from a data-plane perspective," in Proc. ACM MSWiM, Paphos, Cyprus, Oct. 2012, pp. 325-332.

[26] D. Grace and M. Mohorcic, Broadband Communications via High Altitude Platforms. New York: Wiley, 2010.

[27] A. Mohammed, A. Mehmood, F.-N. Pavlidou, and M. Mohorcic, "The role of high-altitude platforms (HAPs) in the global wireless connectivity," Proc. IEEE, vol. 99, no. 11, pp. 1939-1953, Nov. 2011.

[28] F. Wang, J. Xu, X. Wang, and S. Cui, "Joint offloading and computing optimization in wireless powered mobile-edge computing systems," IEEE Trans. Wireless Commun., vol. 17, no. 3, pp. 17841797, Mar. 2018.

[29] S. Bi and Y. J. Zhang, "Computation rate maximization for wireless powered mobile-edge computing with binary computation offloading," IEEE Trans. Wireless Commun., vol. 17, no. 6, pp. 41774190, Jun. 2018.

[30] F. Zhou, Y. Wu, R. Q. Hu, and Y. Qian, "Computation rate maximization in UAV-enabled wireless-powered mobile-edge computing systems," IEEE J. Sel. Areas Commun., vol. 36, no. 9, pp. 19271941, Sep. 2018.

[31] D. Killinger, "Free space optics for laser communication through the air," Opt. Photonics News, vol. 13, no. 10, p. 36, Oct. 2002.

[32] Y. Zeng and R. Zhang, "Energy-efficient UAV communication with trajectory optimization," IEEE Trans. Wireless Commun., vol. 16, no. 6, pp. 3747-3760, Jun. 2017.

[33] L. Xiao, C. Xie, M. Min, and W. Zhuang, "User-centric view of unmanned aerial vehicle transmission against smart attacks," IEEE Trans. Veh. Technol., vol. 67, no. 4, pp. 3420-3430, Apr. 2018.

[34] B. Roberson, "The Colonel Blotto game," Econ. Theory, vol. 29, no. 1, pp. 1-24, Jan. 2006.

[35] S. Hart, "Discrete Colonel Blotto and General Lotto games," Int. J. Game Theory, vol. 36, no. 3-4, pp. 441-460, Oct. 2007.

[36] S. Guan, J. Wang, H. Yao, C. Jiang, Z. Han, and Y. Ren, "Colonel blotto games in network systems: Models, strategies, and applications," IEEE Trans. Netw. Sci. Eng., vol. 7, no. 2, pp. 637-649, Apr. 2020.

[37] A. Ferdowsi, A. Sanjab, W. Saad, and T. Basar, "Generalized colonel blotto game," in Proc. Annual American Control Conference, Milwaukee, WI, Jun. 2018, pp. 5744-5749.

[38] M. Hajimirsadeghi, G. Sridharan, W. Saad, and N. B. Mandayam, "Internetwork dynamic spectrum allocation via a colonel blotto game," in Proc. IEEE CISS, Princeton, NJ, Mar. 2016.
[39] C. Thomas, "N-dimensional blotto game with heterogeneous battlefield values," Econ. Theory, vol. 65, no. 3, pp. 509-544, Jan. 2017.

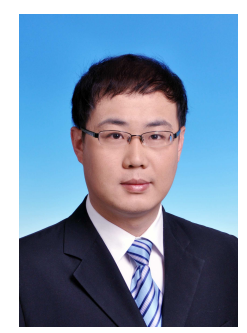

Long Zhang (M'16) received the B.S. degree in communication engineering at the School of Information Engineering from China University of Geosciences, Wuhan, China, in 2006 and the $\mathrm{Ph} . \mathrm{D}$. degree in communication and information systems at the School of Computer and Communication Engineering from University of Science and Technology Beijing, Beijing, China, in 2012. In 2017, he was a Visiting Scholar with the School of Computing, Tokyo Institute of Technology, Tokyo, Japan. From 2018 to 2020, he was a Postdoctoral Fellow with the Department of Electrical and Computer Engineering, University of Houston, Houston, TX, USA. He is currently an Associate Professor with the School of Information and Electrical Engineering, Hebei University of Engineering, Handan, China. His research interests include aerial access networks, fog/edge computing, network functions virtualization, $5 \mathrm{G} / 6 \mathrm{G}$ wireless communications, radio resource allocation, and machine learning.

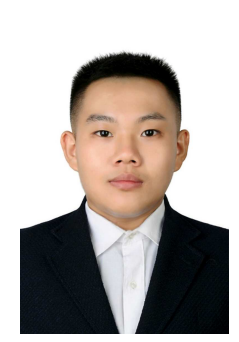

Yao Wang received the B.S. degree in the School of Information Engineering from the Baoding University, Baoding, China in 2019. He is currently working towards the M.S. degree in the computer science and technology at the School of Information and Electrical Engineering, Hebei University of Engineering, Handan, China. His current research interests include $\mathrm{B} 5 \mathrm{G} / 6 \mathrm{G}$ wireless communications, aerial access networks, far-field wireless power transfer, privacy protection, and game theory.

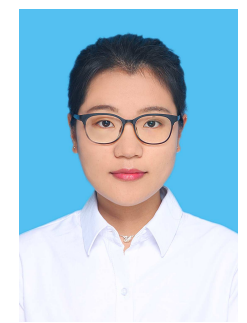

Minghui Min received the B.S. degree in automation from QuFu Normal University, Rizhao, China, in 2013, the M.S. degree in control theory and control engineering from Shenyang Ligong University, joint training with, Shenyang Institute of Automation, Chinese Academy of Sciences, Shengyang, China, in 2016. She received the Ph.D. degree with the Department of Information and Communication Engineering, Xiamen University, Xiamen, China. She was a Visiting Scholar with the Department of Computer Science and Engineering, University of Houston, Houston, USA. She is currently a teacher with the School of Information and Control Engineering, China University of Mining and Technology, Xuzhou, China. Her research interests include network security, privacy preservation, and wireless communications.

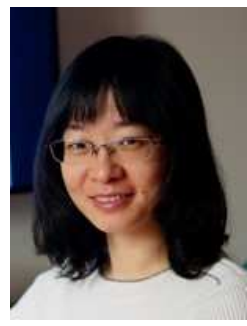

Chao Guo received her B.S. degree from North China Institute of Science and Technology in 2009. She obtained the Ph.D. degree in the Department of Communication Engineering at the School of Computer and Communication Engineering, from University of Science and Technology Beijing in 2015. Now, she is a Lecturer in the Department of Electronic and Communication Engineering, Beijing Electronics Science and Technology Institute, China, and also a Postdoctoral Fellow with the Institute of Information Engineering, Chinese Academy of Sciences. Her research interests include transmission control design, network load balancing, and communications security. 


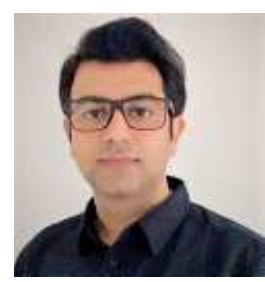

Vishal Sharma (S'13-M'17) received the $\mathrm{PhD}$ and $\mathrm{BTech}$ degrees in computer science and engineering from Thapar University (2016) and Punjab Technical University (2012), respectively. $\mathrm{He}$ is working as a Lecturer ( Assistant Professor) in the School of Electronics, Electrical Engineering and Computer Science (EEECS) at the Queen's University Belfast (QUB), Northern Ireland, United Kingdom. Before coming to QUB, he was a Research Fellow in the Information Systems Technology and Design (ISTD) Pillar at the Singapore University of Technology and Design (SUTD), Singapore where he worked on the future-proof blockchain systems funded by SUTD-MoE. From Nov'16 to Mar'19, he worked in the Information Security Engineering Department at Soonchunhyang University, South Korea in multiple positions (Nov'16 to Dec'17: Postdoctoral Researcher Jan'18 to Mar'19: Research Assistant Professor). He also held a joint postdoctoral position with Soongsil University, South Korea. He was affiliated with the Industry-Academia Cooperation Foundation and the Mobile Internet Security lab at Soonchunhyang University. Before this, he worked as a lecturer in the Computer Science and Engineering Department at Thapar University, India. He is the recipient of three best paper awards from the International Conference on Communication, Management and Information Technology (ICCMIT), Warsaw, Poland, in April 2017; from CISC-S'17 South Korea in June 2017; and from loTaas Taiwan in September 2017. He is the member of IEEE, a professional member of ACM and past Chair for ACM Student Chapter-TIET Patiala. He has authored/co-authored more than 100 journal/conference articles and book chapters and co-edited two books with Springer. $\mathrm{He}$ served/serving as a guest editor of MIS, IJDSN, WCMC, MDPI (Sensors, Drones, Future Internet), and Autosoft journals. He was the track chair of MobiSec'16 and AIMS-FSS'16, and PC member and reviewer of MIST'16 and MIST'17, respectively. He has served/serving as the TPC member of ETIC-2019, WiMO-2019, ITNAC-IEEE TCBD'17, ICCMIT'18, CoCoNet'18, and ITNAC-IEEE TCBD'18. Furthermore, he serves as a reviewer for various ACM/IEEE Transactions and other journals. He also serves as the ATE for the IEEE Communications Magazine and an AE for the IET-CAAI TRIT, Wireless Communications and Mobile Computing, and IET Networks. His areas of research and interests are $5 \mathrm{G}$ networks, Blockchain systems, aerial (UAV) communications, CPSIoT behavior-modeling, and mobile Internet systems.

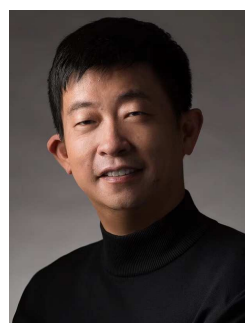

Zhu Han (S'01-M'04-SM'09-F'14) received the B.S. degree in electronic engineering from Tsinghua University, in 1997, and the M.S. and Ph.D. degrees in electrical and computer engineering from the University of Maryland, College Park, in 1999 and 2003, respectively.

From 2000 to 2002, he was an R\&D Engineer of JDSU, Germantown, Maryland. From 2003 to 2006, he was a Research Associate at the University of Maryland. From 2006 to 2008, he was an assistant professor at Boise State University, Idaho. Currently, he is a John and Rebecca Moores Professor in the Electrical and Computer Engineering Department as well as in the Computer Science Department at the University of Houston, Texas. His research interests include wireless resource allocation and management, wireless communications and networking, game theory, big data analysis, security, and smart grid. Dr. Han received an NSF Career Award in 2010, the Fred W. Ellersick Prize of the IEEE Communication Society in 2011, the EURASIP Best Paper Award for the Journal on Advances in Signal Processing in 2015, IEEE Leonard G. Abraham Prize in the field of Communications Systems (best paper award in IEEE JSAC) in 2016, and several best paper awards in IEEE conferences. Dr. Han was an IEEE Communications Society Distinguished Lecturer from 2015-2018, AAAS fellow since 2019 and ACM distinguished Member since 2019. Dr. Han is $1 \%$ highly cited researcher since 2017 according to Web of Science. Dr. Han is also the winner of 2021 IEEE Kiyo Tomiyasu Award, for outstanding early to mid-career contributions to technologies holding the promise of innovative applications, with the following citation: "for contributions to game theory and distributed management of autonomous communication networks." 